\title{
Voir une chenille, dessiner un serpent à plumes. Une relecture analogique de l'hybridité et des êtres imaginaires en Mésoamérique préhispanique
}

Seeing a caterpillar, drawing a feathered serpent. An analogical reinterpretation of hybridity and imaginary beings in pre-Columbian Mesoamerica Ver una oruga, dibujar una serpiente emplumada. Una relectura analógica de la hibridez y de los seres imaginarios en la Mesoamérica prehispánica

Dimitri Karadimas

\section{OpenEdition \\ Journals}

Édition électronique

URL : https://journals.openedition.org/jsa/13673

DOI : 10.4000/jsa. 13673

ISSN : 1957-7842

\section{Éditeur}

Société des américanistes

Édition imprimée

Date de publication : 17 septembre 2014

Pagination : 7-43

ISSN : 0037-9174

Référence électronique

Dimitri Karadimas, « Voir une chenille, dessiner un serpent à plumes. Une relecture analogique de l'hybridité et des êtres imaginaires en Mésoamérique préhispanique », Journal de la Société des américanistes [En ligne], 100-1 | 2014, mis en ligne le 01 janvier 2016, consulté le 02 septembre 2022. URL : http://journals.openedition.org/jsa/13673 ; DOI : https://doi.org/10.4000/jsa. 13673 


\title{
VOIR UNE CHENILLE, DESSINER UN SERPENT À PLUMES. UNE RELECTURE ANALOGIQUE DE L'HYBRIDITÉ ET DES ÊTRES IMAGINAIRES EN MÉSOAMÉRIQUE PRÉHISPANIQUE
}

\author{
Dimitri KARADIMAS *
}

En prenant le parti d'analyser les êtres imaginaires et les figurations d'hybrides en Mésoamérique comme des retranscriptions imagées d'êtres réels selon des principes de l'analogie formelle, cette contribution se propose de revenir sur la figure de Quetzalcóatl, tant dans l'iconographie de Teotihuacan que dans celle, plus tardive, des Aztèques. Après avoir passé en revue certains principes de la perception intervenant dans le mode de nomination des espèces, leur application à des matériaux iconographiques mettant en combinaison des êtres - ou des parties d'êtres - distincts permet de rendre compte de leur présence concomitante sur une seule coiffe ou en tant qu'être mythologique. [Mots-clés : Aztèques, Teotihuacan, Quetzalcóatl, êtres imaginaires, analogisme, iconographie, lépidoptères.]

Seeing a caterpillar, drawing a feathered serpent. An analogical reinterpretation of hybridity and imaginary beings in pre-Columbian Mesoamerica. This contribution suggests to look at the figure of Quetzalcoatl - in the Teotihuacan iconography as in the later Aztec one - by analyzing imaginary beings and the representations of hybrids in Mesoamerica as an analogical transcription of real beings according to the principles of formal analogy. After going through a review of the perception processes entering in the way the names of species are built in natural languages, we apply these processes to iconographical materials that combines distinct beings - or parts of beings -, this enables us to interpret their occurrences on feather headdresses or as mythological figures. [Key words: Aztecs, Teotihuacan, Quetzalcoatl, imaginary beings, analogism, iconography, butterflies.]

Ver una oruga, dibujar una serpiente emplumada. Una relectura analógica de la hibridez $y$ de los seres imaginarios en la Mesoamérica prehispánica. El propósito de esta contribución es de mirar la figura precolombina de Quetzalcóatl - tanto en la

* Laboratoire d'anthropologie sociale, 52 rue du Cardinal-Lemoine, 75005 Paris [dimitri.karadimas@college-de-france.fr].

Journal de la Société des Américanistes, 2014, 100-1, pp. 7-43. (C) Société des Américanistes. 
iconografía de Teotihuacan que en la de los más tardíos aztecas - como una reelaboración analógica de seres reales (principalmente orugas). Esto se hace gracias a un análisis de los seres imaginarios y híbridos de Mesoamérica, interpretándolos como figuraciones evocativas de animales existentes. Después de dar una descripción de los procesos de percepción que entran en la manera de nombrar a las especies en las lenguas naturales, se aplica estos procesos a un material iconográfico que mezcla a seres - o partes de seres - distintos, permitiendo así interpretar la presencia de estos últimos ya sea en penachos de plumas o como seres mitológicos. [Palabras claves: aztecas, Teotihuacán, Quetzalcóatl, seres imaginarios, analogismo, iconografía, mariposas.]

Comme la plupart des aires culturelles à travers le monde, la Mésoamérique a développé, dans sa mythologie et dans son iconographie, des figures d'êtres imaginaires et d'hybrides, la plus connue étant celle du "serpent à plumes ». Ces figures ont été l'objet d'interprétations menées par différentes disciplines, dont l'anthropologie et l'archéologie, qui y voyaient des indices tangibles de «croyances » et de constitution de déités. Partageant ce trait culturel avec d'autres civilisations comme la Grèce classique, qui nous est en apparence plus familière, le statut de l'hybride n'est que rarement questionné en termes de ressemblance avec des êtres existant dans l'environnement.

L'hybridité, en effet, dans son sens le plus général, c'est-à-dire comme un mélange d'éléments non issus d'un même organisme mais une combinaison de parties de plusieurs espèces pour en former une nouvelle, pose la question des ontologies dans chaque système culturel donné. Parmi les hybrides, les êtres composites forment une catégorie particulière, le plus souvent objets de croyances ou, du moins, catalogués comme tels. Les êtres mythologiques font partie de cette catégorie. Soit ils sont composés d'une pluralité de parties distinctes n'appartenant pas à la même espèce, soit ils résultent d'une combinaison qui n'existe pas dans le monde réel, raison pour laquelle ils sont le plus souvent rangés sous l'appellation d'« êtres imaginaires ».

Au-delà de la saisie du vivant par les sens, la constitution des êtres composites repose avant tout sur les facultés de perception et de nomination. Nous avons déjà montré comment opérait l'esprit humain, toutes cultures confondues, pour former ces êtres (Karadimas 2010). Si, dans tous les systèmes de nomenclatures issus des langues humaines, les noms composés d'espèces réelles sont formés par l'agrégation des noms de deux objets ou espèces distinctes, il en va de même pour la création des êtres imaginaires.

Cette contribution se propose, en premier lieu, de décrire cette opération mentale pour esquisser ensuite une tentative d'application à des exemples mésoaméricains. Notre propos est le suivant : premièrement, reposer la question de la place des principes de perception dans la description des êtres qui peuplent l'environnement; deuxièmement, nous interroger sur l'interaction 
entre description et figuration ; enfin, envisager la catégorie de croyance (plus particulièrement religieuse) à la lumière des principes ici dégagés.

\section{LIRE L'ENVIRONNEMENT}

Sous leurs formes picturales ou narratives, les monstres et les êtres imaginaires sont, le plus souvent, le résultat d'une recombinaison graphique des saillances visuelles que chaque espèce, chose, voire item, produit sur un observateur humain dans une culture donnée. Ces « saillances » sont des éléments singuliers possédés par ces items, que les systèmes culturels ont maintenus nominalement pour les caractériser. Parler de " poisson-scie », c'est faire référence à la singularité de ce poisson d'avoir un rostre qui rappelle, pour notre culture, un outil. Il s'agit, avant tout, d'un processus mental, puis d'une inscription de la saillance perçue dans la langue. Appliquée à du matériau iconographique, cette faculté de reconnaissance ouvre des possibilités d'interprétations plus amples que d'autres méthodes, dans la mesure où tout élément perçu est susceptible de générer d'autres images mentales que sa simple identification en tant que taxon ou item. Dans cette perspective, il nous aurait été possible d'avoir recours à la tripartition panofskienne de l'analyse iconographique tant utilisée par l'histoire de l'art. Celle-ci postule trois étapes dans sa démarche: la description préiconographique - qui ne donnerait qu'un rendu des formes - ; la description iconographique - de reconnaissance des personnages et/ou des identités en général - ; et, enfin, le moment de l'interprétation iconologique, c'est-à-dire du résultat combiné des différents éléments dans un ensemble pictural donné (tableau, scène murale, etc.) pour en révéler le sens culturel. Le principal problème de cette approche tient au fait que la description des formes (le niveau pré-iconographique) est déjà, à tout le moins pour les sciences cognitives, un type d'interprétation puisque l'univers du perceptible est infiniment plus vaste que ce qui est identifié. Savoir si cette forme d'interprétation est culturellement déterminée reste un débat ouvert. Il demeure que percevoir, c'est déjà discriminer et, partant, interpréter : il n'existe, en quelque sorte, pas de perception « pure », si ce n'est médiatisée par les connaissances individuelles (elles-mêmes socialisées, c'est-à-dire incluses dans un processus culturel).

Il nous faut ainsi admettre que la description implique un certain regard. Ce que nous proposons est une tentative de reconstruction du regard que les Amérindiens de la Mésoamérique préhispanique portaient sur le monde par l'intermédiaire de leurs différentes expressions iconographiques. Si le sens de ces images créées à une époque révolue est rendu opaque par l'absence d'écrits ou d'exégèses explicites, sa saisie reste possible dans la mesure où les êtres qui y sont représentés forment, le plus souvent, des combinés visuels auxquels les modalités de nominations dans les langues naturelles de la région nous permettent d'avoir accès. 
Ainsi, les processus de nomination des espèces font intervenir, en quelque culture que ce soit, des principes de hiérarchisation taxonomique. Le niveau qui nous occupe est celui de l'espèce et de son identification lorsqu'elle est désignée par un nom composé. Ce nom implique souvent un genre (ou taxon : « cheval », « serpent », " mouche », etc.) qui se voit associé à une caractéristique ou un trait particulier. Ce trait peut être soit une couleur («mouche-verte»), soit un état ( " chien d'arrêt »), soit une référence à un milieu ou à domaine sur - ou dans lequel vit l'espèce («araignée d'eau », «tigre du poirier»-qui n'est pas un félin...). Le plus souvent pourtant, il s'agit d'une caractéristique - les psychologues de la perception diraient une « saillance »- qui rappelle une autre forme possédée par ou appartenant à une autre espèce. Le nom de cette dernière se trouve ainsi associé au genre pour caractériser l'espèce. Dans une précédente contribution (Karadimas 2010), nous avions déjà pris l'exemple du poisson-chat pour montrer que les vibrisses étaient une singularité de cette espèce parmi la classe des poissons et que l'analogie formelle la plus approchante était à rechercher dans une autre espèce, le chat, qui possède des organes sensitifs analogues. La forme nominale finale " poisson-chat » est ainsi un raccourci, cristallisé dans les connaissances encyclopédiques d'une culture donnée, qui ne demande pas constamment d'être analysé par les locuteurs naturels d'une langue. Ce principe est valable dans toutes les langues et cultures.

Le domaine de la figuration est plus problématique. En effet, plusieurs possibilités s'offrent aux artistes des différents systèmes culturels. La solution la plus répandue est de figurer le plus fidèlement possible l'espèce en question. Pour le cas $\mathrm{du}$ poisson-chat, cela consiste à en faire un portrait naturaliste qui place cette espèce dans la situation d'être équivalente à une individualité. Une autre solution est de produire un être combiné qui reprend visuellement les composantes de son nom (issues, il faut le rappeler, de saillances perceptives). Pour le poisson-chat, c'est réaliser l'image d'un poisson avec une tête de chat. Cette solution lève l'ambigüité de la figuration d'une espèce traitée comme individualité (ou portrait naturaliste), puisqu'elle en appelle au système de dénomination des espèces plutôt qu'à leur image. L'image de l'hybride accorde plutôt une place à la connaissance encyclopédique en demandant au spectateur de déchiffrer la figuration, malgré le fait que l'être composite qui en résulte est en apparence fantastique pour nos traditions « naturalistes » (dans le sens que Descola 2005 donne à ce système ontologique). Cela tient au fait que notre tradition accorde à la figuration naturaliste une primauté (ethnocentrique ?) sur l'ensemble des autres modalités figuratives. Face à des images d'êtres composites, il faut donc commencer par évacuer la possibilité d'une figuration d'espèces réelles avant d'envisager de les considérer comme des portraits d'êtres surnaturels, mythologiques et/ou des figures religieuses. Or ce point mérite d'être tout particulièrement souligné pour la Mésoamérique qui a vu fleurir plusieurs systèmes d'écritures pictographiques, aussi bien celui, idéographique, des Aztèques que celui, logo-syllabique, des Mayas. 
Ainsi, pour le cas de la figuration d'animaux à noms composés, il est possible de montrer qu'au moment du contact avec le monde hispanique et l'arrivée d'une écriture entièrement phonétique (celle des Mayas ne l'était que partiellement), la Mésoamérique n'en avait pas moins gardé, avec les lettrés aztèques, une forte composante analogiste dans son mode de figuration. Dans la Figure 1, tirée du Códice Florentino (1979), quatre espèces de poissons sont représentées par les composantes visuelles qui forment leurs noms en nahuatl : "poisson-hibou », « poisson-colibri », " poisson-papillon » et «poisson-jaguar ». Or, comme l'a signalé López Luján (1991, pp. 247-248) « l'artiste était plus soucieux d'illustrer le nom en nahuatl que de représenter les animaux tels qu'ils apparaissent ». La figuration «fonctionne » ici cognitivement à la façon d'un glyphe, ce que plusieurs auteurs ont déjà souligné. Ce qui n'a peut-être pas été suffisamment rappelé est l'implication équivalente et inverse de ce résultat figuratif : l'environnement et donc chacune des espèces naturelles qui le composent sont des objets susceptibles d'être «lus» ou, pour le moins, de subir une tentative de déchiffrement. Les phénomènes de la lecture et de l'écriture ne sont, de la sorte, qu'une variante de recréation humaine d'une forme rencontrée dans l'environnement et donc susceptible, comme sa forme naturelle, d'être « lue » (c'est-à-dire reconnue).

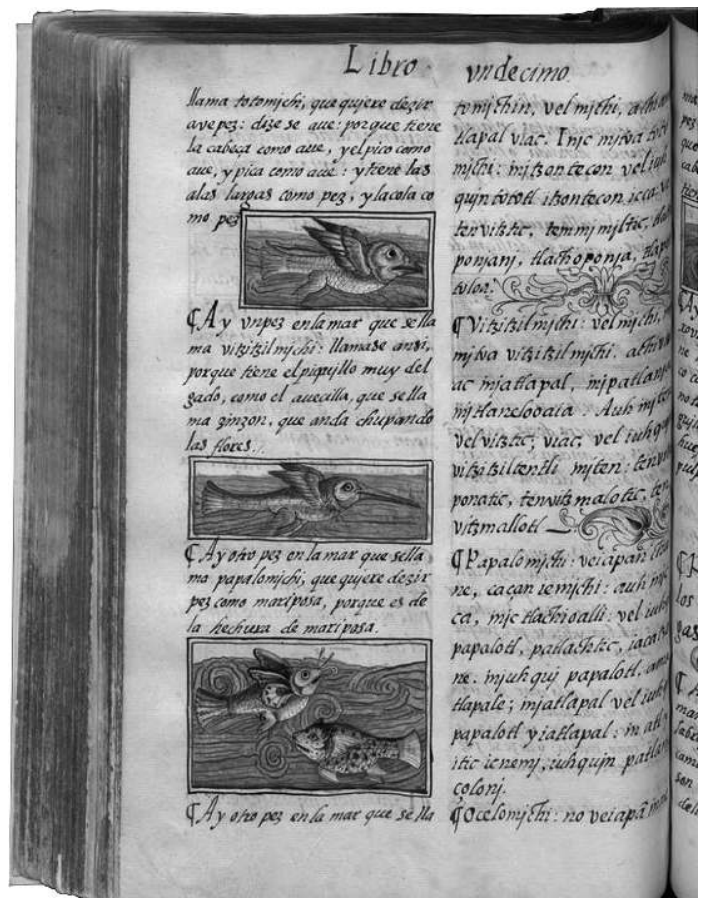

FIG. 1 - Quatre poissons figurés par une combinaison des êtres entrant dans leurs noms composés Códice Florentino (1979, XI, folio 62v). 
Chaque espèce est potentiellement détentrice d'une ou de plusieurs caractéristiques autres que celles qui marquent son genre: si toutes les mouches se ressemblent, seules quelques-unes sont de couleur vert métallique. Si tous les poissons possèdent, avec des degrés divers, un même aspect général, ce qui produit leur singularité renvoie mentalement l'observateur à un autre registre que celui du poisson. Ce « double possédé en image ${ }^{1}$ marque ainsi l'individualité de l'espèce dans une famille ou un genre, c'est-à-dire une opération analogue à celle qu'avait déjà soulignée Lévi-Strauss dans La Pensée sauvage (1963). « Lire » l'environnement implique ainsi d'en faire ressortir les formes significatives, c'est-à-dire de produire des énoncés « gestaltistes ", à base d'icônes (dans le sens de la sémiologie de Peirce, à savoir un signe qui acquiert sa signification par ressemblance avec l'objet). Avant d'examiner quelques cas particuliers, il convient de souligner que toute génération d'images mentales n'a pas vocation à être inscrite dans une forme lexicalisée dont la plus commune serait le nom composé. Certaines analogies formelles peuvent être rendues dans d'autres situations, comme la figuration, sans que cela n'entraîne une forme nominale propre à la connaissance encyclopédique du système culturel donné.

Dans les actions rituelles enfin, une mise en situation de ces formes mentales peut déboucher sur une scénographie particulière, qui implique autant les animaux que les artéfacts issus des saillances perceptives. Par exemple, s'il fallait faire intervenir le personnage du « poisson-jaguar » dans une séquence rituelle donnée, le tableau scénographique qui en résulterait pourrait être celui d'un combiné entre une forme ichtyologique revêtue par un danseur, à laquelle des traits de jaguar - gueule dotée de crocs, ou tout autre saillance propre à ce félidé, avec une nette préférence pour la peau tachetée ou des vibrisses marquées seraient adjoints. Pour un observateur extérieur, voire pour un spectateur de la culture donnée, le combiné rituel qui en résulte n'est pas accessible de premier abord. Il demande un déchiffrement similaire à celui produit lors de la perception analogique de l'environnement.

Dans les lignes qui suivent, on tentera d'analyser un être fantastique commun à la Mésoamérique, le "serpent à plumes », aussi nommé Quetzalcóatl, en accordant une préférence à une approche qui tient compte des modalités cognitives présentes dans la création d'êtres composites, notamment par la considération des facultés de reconnaissance et de description d'êtres réels. Une fois recombiné, l'ensemble des images issues de ces mécanismes perceptifs forme un être étrange, hybride, mais n'étant pas pour autant le résultat d'un arbitraire culturel.

Il nous faut, comme premier pas, faire un détour par l'analyse de deux motifs communs dans cette aire culturelle, celui du papillon et de sa combinaison avec le félin. 


\section{Des papillons en Mésoamérique indienne}

Nous choisirons, dans un premier temps, de prendre en considération l'univers des lépidoptères et leur mode de figuration dans l'espace de la Mésoamérique, non pas par simple penchant « entomophile », mais parce que plusieurs cultures de cette aire, en particulier celle de Teotihuacan, leur ont accordé une place importante dans leur art. Si nous ne pouvons être catégoriques sur les motivations qui ont poussé des peuples de Mésoamérique à choisir les papillons, parmi l'ensemble des animaux présents dans leur environnement, pour évoquer une partie du destin post-mortem de leurs guerriers (surtout les Aztèques), il faut tout de même remarquer que le processus de métamorphose complète que subit la chenille pour prendre sa forme adulte a souvent été repris, comme dans la Grèce ancienne, pour signifier la vie post-mortem d'une des composantes de la personne humaine. Ainsi, les guerriers aztèques morts au combat accompagnaient le soleil sous la forme de papillons alors que leur dépouille corporelle restait sur un plan terrestre ( $c f$. Ragot 2000) et nombre de déités mésoaméricaines possèdent une forme de lépidoptère dont l'origine se trouve certainement dans la culture de Teotihuacan: "The numerous ceramic objects in Teotihuacan-style found in southern Guatemala suggest that a militaristic butterfly deity was the predominant cult figure from the Teotihuacan pantheon that Teotihuacan warriors and merchants took aboard with them " (Berlo 1983, p. 95).

Nous utiliserons comme principale source bibliographique l'ouvrage de Beutelspacher Las mariposas entre los antiguos mexicanos (1988), qui passe en revue, de façon quasi exhaustive, aussi bien les écrits des premiers chroniqueurs espagnols que les formes graphiques qui sont données aux papillons sur les monuments, les artéfacts et dans les codex écrits par les Indiens. Nous avons aussi largement puisé dans l'immense stock de données présentées sur le site internet de la Foundation for the Advancement of Mesoamerican Studies (FAMSI) et, en particulier, le Catalogue of Zapotec effigy vessels de Sellen ( $c f$. aussi Sellen 2007). Enfin, nous reprendrons plusieurs données publiées par Taube (2000) concernant l'iconographie des serpents fantastiques de la Mésoamérique.

Commençons par prendre en considération des formes attestées de noms composés. Certains papillons, comme ceux de la famille des Caligo ou des Automeris ( $c f$. infra), possèdent des ocelles qui rappellent des yeux de chouettes. Dans la langue nahuatl, l'association avec la chouette se retrouve dans le nom du papillon Xochiautecolotl : "Xóchiautecolotl, según Simeón (1977), se compone de las siguientes raíces: xóchitl, flor, áhuatl, espina, y tecólotl, tecolote, búho, lechuza, pudiendo significar esta última palabra "que vive sobre los árboles" " (Beutelspacher 1988, p. 22). 
Plutôt que de considérer que tecólotl (« hibou») fait référence au fait de « vivre sur les arbres », comme le propose Beutelspacher, il est possible de pencher vers une interprétation de l'aspect du papillon : en combinaison avec les ailes antérieures, les ocelles qu'il porte sur les ailes postérieures évoquent, pour un observateur extérieur, l'image d'une face de chouette. C'est en effet en imitant les yeux d'un prédateur comme la chouette que les papillons font fuir les lézards ou d'autres oiseaux eux-mêmes victimes des chouettes : cette forme mimétique les fait, le plus souvent, nommer à partir d'un combiné du type « papillon-chouette ».

Dans la culture zapotèque, contemporaine de celle de Teotihuacan, les coiffes de certaines figures en terre cuite (Figure 2) montrent que cette thématique fut reprise dans l'iconographie. Y apparaissent autant l'oiseau de nuit - reconnaissable à son bec - que le papillon, suivant une imbrication respectant la disposition du motif au sein des ailes du lépidoptère : l'ensemble s'inscrit dans une coiffe de plumes qui couvrait la tête humaine des vases effigies.

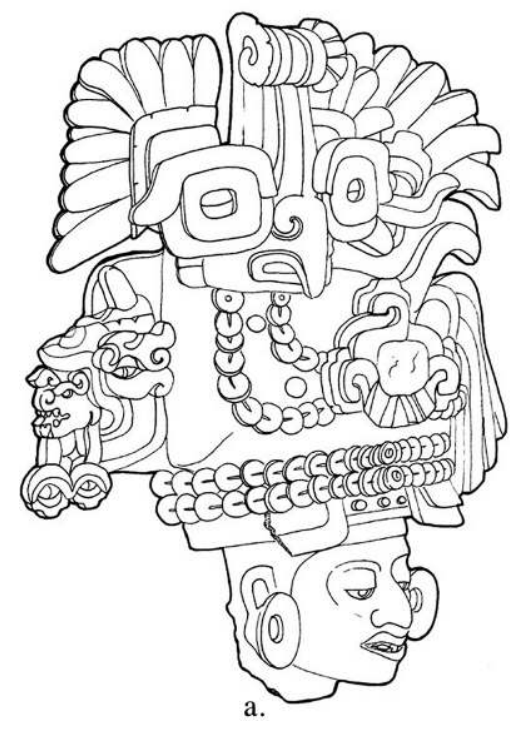

FIG. 2a et b - Coiffe papillon à la face de chouette [culture zapotèque, Ejutla, Oaxaca, Buffalo Museum of Science, C 15997/Acc. 47151, A. Sellen sur site FAMSI] et papillon de nuit Automeris aux yeux de chouettes [C2006-2014 bugalirious-STOCK].

Il est possible d'interpréter cette modalité figurative zapotèque en reprenant la constatation de Berlo (voir supra) selon laquelle beaucoup des formes de lépidoptères rencontrées dans les expressions artistiques des différentes cultures mésoaméricaines eurent leur origine dans l'art de Teotihuacan, où elles étaient rendues, le plus souvent, de façon figurative. 
Dans la Figure 3, les antennes, les yeux et la trompe sont rendus par des compositions de plumes. Les yeux du papillon sont marqués par des plumes concentriques, placées de part et d'autre de sa trompe ou proboscis. Celle-ci, également marquée par des plumes, occupe, sous sa forme enroulée ou en spirale, une place médiane. Les antennes partent de chaque côté et, courbées, se terminent par des plumeaux. Les deux paires d'ailes sont en position inversée, les antérieures au-dessus dela composition et la pointe desailes postérieures visibleentreles yeux et les antennes. Cette disposition est celle d'un papillon plongeant en vue dorsale.

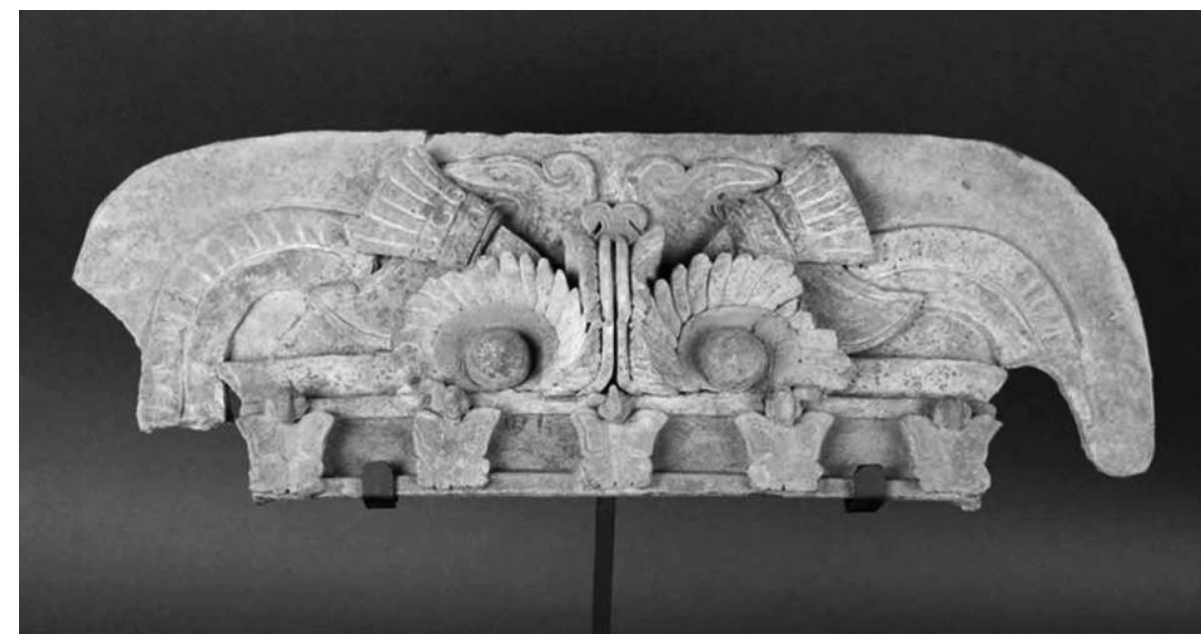

FIG. 3 - Coiffe de la partie supérieure d'un couvercle d'encensoir de style teotihuacan [Museo chileno de arte precolombino $\mathrm{n}^{\circ} 0111$, Santiago, Chili].

Franco (1955) a montré que les principes de composition formelle des lépidoptères à Teotihuacan sont analogues à ceux rencontrés dans d'autres iconographies mésoaméricaines. Parmi les espèces retenues, plusieurs appartiennent à la famille des Papilio. C'est en particulier le cas des variétés de multicaudatus, également connues comme « porte-queues » en français, en raison de la particularité du dessin de leurs ailes postérieures qui portent plusieurs échancrures et excroissances (les « queues » présentes dans le nom composé). Pour les Aztèques, cette caractéristique permettait de les associer au feu, par l'analogie qu'entretiennent ces excroissances avec l'image des flammes et flammèches d'un brasier.

Dans les compositions de la culture zapotèque, il semble que, lorsque la combinaison doit rendre compte de l'espèce " papillon-chouette », elle y intègre les ocelles en forme d'œil de rapace, tout en ajoutant un bec ouvert à la place qu'occuperait cet organe au sein d'une tête de hibou. L'ensemble nous apparaît quelque peu étrange, mais ne fait que reprendre les images mentales que la 
perception de l'espèce-source a produites. Dans les figurines en terre cuite, l'être qui porte cette coiffe se revêt ainsi des images d'un « papillon-chouette » et/ou d'un « papillon-rapace ». En effet, tous les ocelles portés sur les ailes de papillons ne renvoient pas à la seule famille des Strigidés (chouettes et hiboux). Certains peuvent être associés aux yeux d'autres volatiles, soit à ceux des oiseaux de proie en général, soit à ceux d'espèces moins facilement reconnaissables comme les chauves-souris, voire, dans certaines variétés d'Automeris, à ceux des félins (comme le puma, mais il s'agit là d'une autre discussion). Dans les compositions issues de la culture de Teotihuacan, par exemple, même si les couleurs des plumes renvoient en partie à l'oiseau quetzal, c'est aussi aux yeux et au regard de l'oiseau de proie nocturne que les ocelles ont été associés, pour réaliser un combiné imagé $\mathrm{du}$ 《papillon à face de chouette» ou «papillon aux yeux de chouette» (cf. Figure 4). Sur ces compositions, en effet, le bec de l'oiseau est celui d'un rapace nocturne et non celui d'un quetzal ${ }^{2}$. Il faudrait ainsi considérer ces compositions comme une mise en image d'une saillance perceptive (qui se manifeste, ou non, dans un nom composé, comme dans la langue nahuatl).

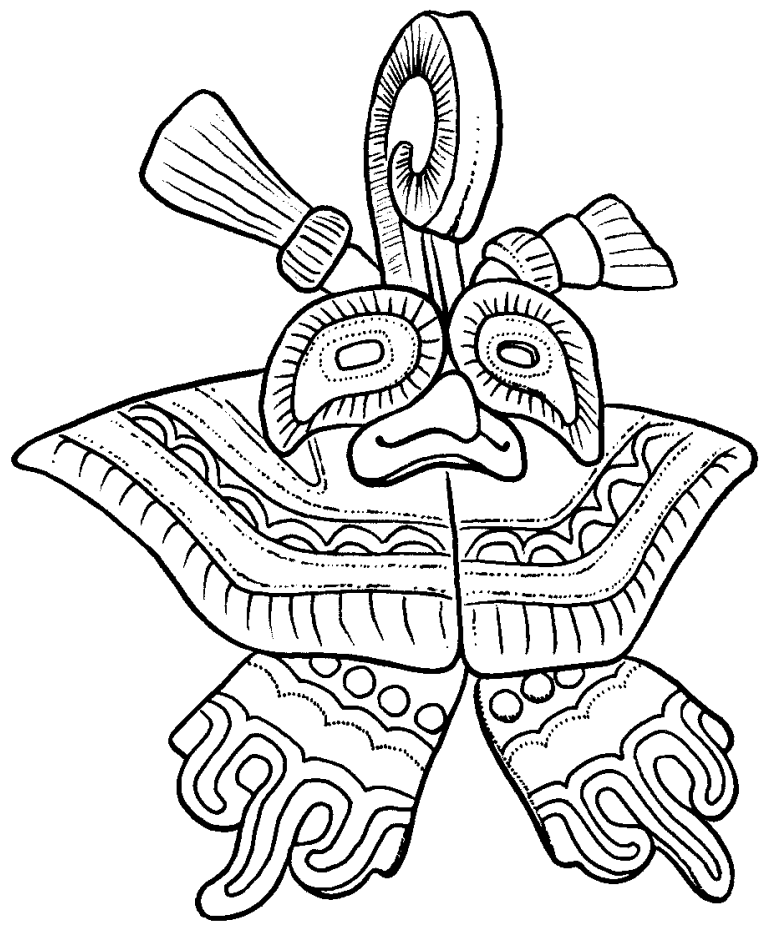

FIG. 4 - Applique en forme de papillon à la face d'oiseau de nuit ou de rapace provenant d'un encensoir-théâtre teotihuacan [Instituto Nacional de Antropología e Historia/Museo Nacional de Antropología, Mexico, 10-80439; dessin N. Latsanopoulos]. 
La combinaison « oiseau-papillon » n'est donc pas issue d'une association arbitraire. Elle n'est pas non plus le fruit d'une volonté d'exprimer, par leur combinaison en une forme hybride, un condensé de deux espaces ou de deux milieux distincts, voire de la relation prédateur/proie qui pourrait être postulée entre, d'un côté, le papillon et, de l'autre, le petit rapace ou l'oiseau nocturne (ce type d'association, déjà présenté par Séjourné pour la culture de Teotihuacan, s'est maintenu ensuite dans plusieurs interprétations iconographiques concernant la Mésoamérique précolombienne ; $c f$. Séjourné 1962, p. 23). Il est plus probable que les artistes zapotèques et de Teotihuacan aient voulu rendre compte de la perception analogique qu'il est possible d'avoir du papillon Automeris et du motif inscrit dans ses ailes. Le fait de savoir quelle fin étaient censées posséder ces coiffes pour la personne qui les portait et de connaître la fonction des figurines dans le système culturel donné est un débat que nous ne mènerons pas ici. Le point qu'il nous faut souligner est que la figure de l'hybride (animal ou composition-cible) résulte d'une perception qui donne sens à certains motifs présents sur l'espèce source. Restons dans cette tradition pour voir si une autre variation ne pourrait pas être trouvée dans ce même registre, et pour montrer que c'est bien cette hypothèse qui prime sur les autres.

Au même titre que certains papillons portent des motifs ocellés qui renvoient à des faces de chouettes, d'autres espèces exploitent une ressemblance avec des faces de félins, voire imitent les tâches blanches présentes sur l'arrière de leurs oreilles. Or c'est précisément ce deuxième type de composition qui se trouve combiné à la structure d'un papillon pour servir de motif de coiffes sur certaines poteries zapotèques. Dans cet art, la combinaison des deux êtres suit celle inscrite dans l'espèce naturelle, rendant ainsi visible une perception qu'en ont eue les Indiens de cette époque, à l'identique de la nôtre (Figure 5).

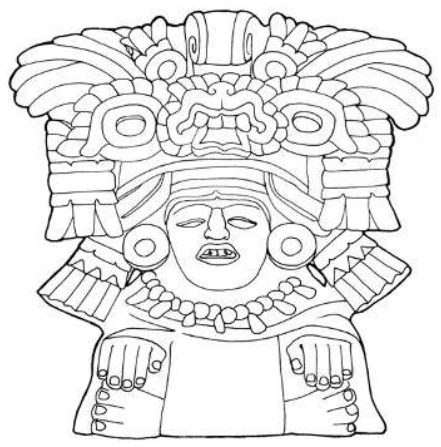

a.

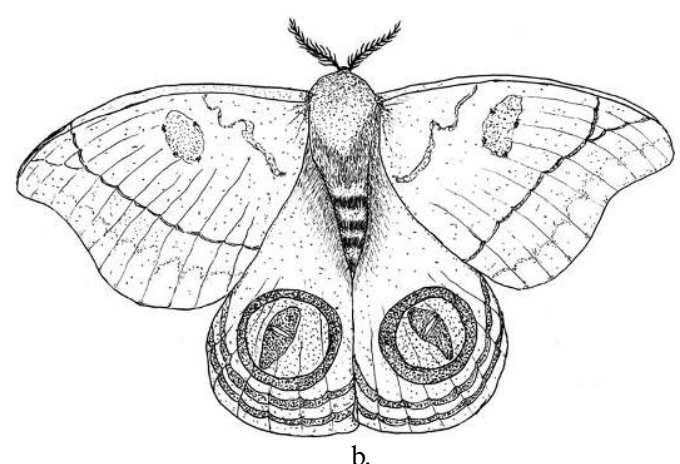

b.

FIG. 5a et $\mathrm{b}$ - Coiffe papillon à face de jaguar [poterie zapotèque, provenance inconnue, Princeton Art Museum, Princeton, New Jersey, A. Sellen sur site FAMSI] et variété d'Automeris aux ocelles en forme d'yeux de félins (c'est-à-dire aux pupilles longitudinales) [dessin de l'auteur D. K]. 
Sur la Figure 6, nous avons associé le papillon porte-queue ( Papilio sp.) et les motifs de face de félins. Sur la surface des ailes antérieures apparaissent des taches et des marbrures qui imitent la forme des yeux de félins, le reste des motifs évoquant celle des oreilles. Sur l'espèce naturelle, l'effet mimétique procède par mise à plat, puisqu'il s'agit d'une image qui est renvoyée, à la manière d'une surface de miroir. Dans ce motif, les papillons font coïncider ${ }^{3}$ leur tête et leurs deux yeux avec le nez ou truffe du félin. Au même titre que, sur les « papillons-chouettes », l'abdomen évoque le bec des rapaces nocturnes, les " papillons-jaguars » font se chevaucher leur corps, thorax et abdomen, avec une gueule ouverte de félin dans laquelle se trouve une langue qui pend. L'imitation est poussée jusqu'à faire coïncider les nervures des ailes postérieures du papillon avec les vibrisses du félin. Cette façon d'imiter une tête de petit félin, ocelot ou margay, fait que le papillon dans son entier correspond à une tête de félidé. On voit donc que les combinaisons jaguar/papillon et rapace/papillon présentes dans l'art mésoaméricain résultent d'une perception qui, suivant que l'une ou l'autre des espèces est prise en compte, produit à la fois un être réel et l'image d'une autre espèce.
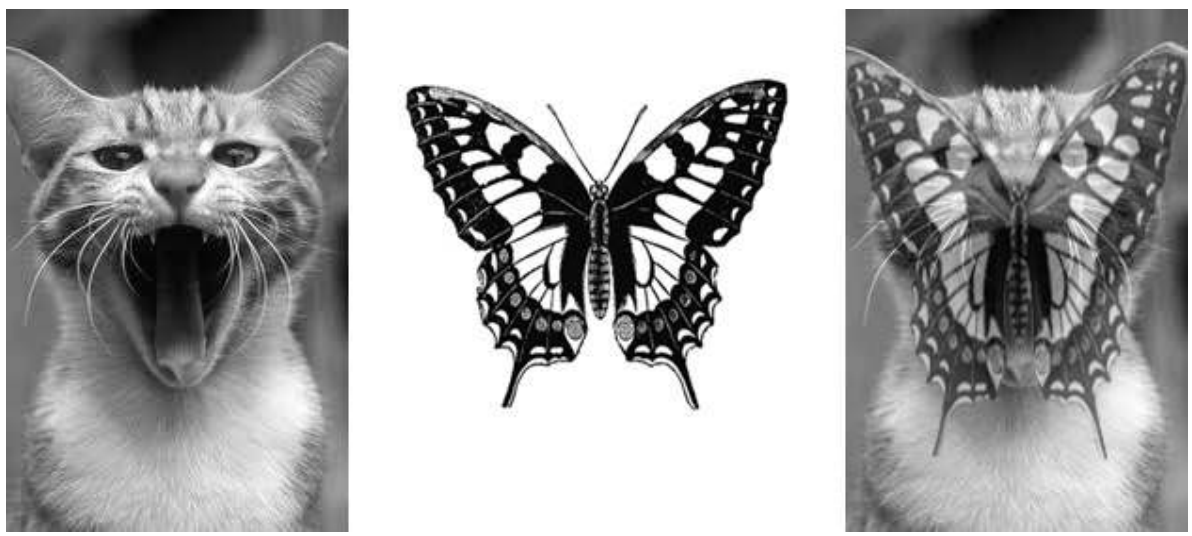

FIG. 6 - Superposition d'un papillon porte-queue Papilio $s p$. sur la face d'un chat afin de souligner le «masque» de félin présent en tant que motif cryptique sur les ailes du lépidoptère [d'après : http://www.petuniversity.com/cats/grooming/teeth-brushing.htm et http://etc.usf. edu/clipart/52300/52302/52302_swallow_tail.htm].

Dans la culture de Teotihuacan, l'utilisation du motif ne suit pas entièrement les mêmes règles (bien que Latsanopoulos nous ait indiqué une configuration analogue dans cet $\operatorname{art}^{4}$ ), puisque le motif mimétique n'est pas traité en relief, comme dans le cas zapotèque, mais «à plat» dans une peinture murale. En revanche, les artistes teotihuacan rendent cette même combinaison en la figurant 
telle qu'elle apparaît sur les papillons. En d'autres termes, même s'ils gardent le motif «à plat », ils reconnaissent les images qui font référence aux félins que laissent apparaître les processus mimétiques sur les ailes des papillons.

Cet ensemble de constatations devrait être pris en compte dans la répartition « guerriers-aigles », « guerriers-jaguars » comme ordres militaires dans la culture aztèque, voire maya, et leur association avec les destins post-mortem sous forme de papillon et d'oiseaux accompagnant le Soleil (cf. Ragot 2000, pp. 172-176). C'est surtout par l'intermédiaire de leur inscription comme motif dans une coiffe que cette thématique apparaît clairement. Pour la compréhension du cas teotihuacan et de la place occupée par la thématique du papillon, il faudrait passer par l'étude des trois autres formes qui lui sont associées, le jaguar réticulé, le serpent à plumes et la conque emplumée. Nous n'aborderons ici que les deux premières formes.

\section{LE FÉLIN À LA COIFFE ET LE JAGUAR RÉTICULÉ DANS L'ART TEOTIHUACAN}

Le félin à la coiffe, ou félin divinisé, est présent, avec le coyote à la coiffe, sur les peintures murales de plusieurs édifices du site de Teotihuacan. La combinaison du félin entier, avec la coiffe qui recouvre sa tête, semble être un indicateur du caractère divin qui lui est accordé. Son corps est également intégralement rehaussé de plumes à la périphérie et d'entrelacements de cordes (recensés dans le Conjunto del Sol, portiques 3 et 13 et le Conjunto de los Jaguares, portique 10 et pièce 10 ; en posture anthropomorphe à Tetitla, pièce et corridor 12 et à Atetelco, Patio Norte, portique 2). Notons dès maintenant que cet entrelacement de fils est une thématique qui peut appartenir à d'autres êtres qui produisent des fils de soie, comme les araignées ou les chenilles lorsqu'elles tissent un cocon. Ce félin réticulé possède une coiffe caractéristique qui le relie aux personnages anthropomorphes de la statuaire teotihuacan. Sa langue bifide est un autre caractère de son anatomie qui apparaît sur différentes représentations qui associent la plumasserie des coiffes à une tête de jaguar de laquelle pend une langue ophidienne (Figure 7). À l'intérieur de sa gueule, apparaît parfois la tête d'un personnage qui a été interprété comme celui de Quetzalcóatl (Séjourné 1962, p. 129, fig. 153).

Associée à deux autres animaux - l'oiseau, par la plume, et le jaguar, par la gueule et les pattes griffues -, cette langue bifide de serpent représente, pour Séjourné, le troisième élément, chtonien, qu'il faut associer à l'air et à la terre. L'animal composite deviendrait, de la sorte, une concaténation de ces trois espaces en un hybride emblématique (ibid., p. 128). Avant de maintenir cette hypothèse, il est nécessaire de constater que cette modalité d'association reprend certains traits déjà présents dans les figurations des papillons dans l'art de Teotihuacan. Si jaguar et plumes sont associés, c'est que celles-ci sont données en 


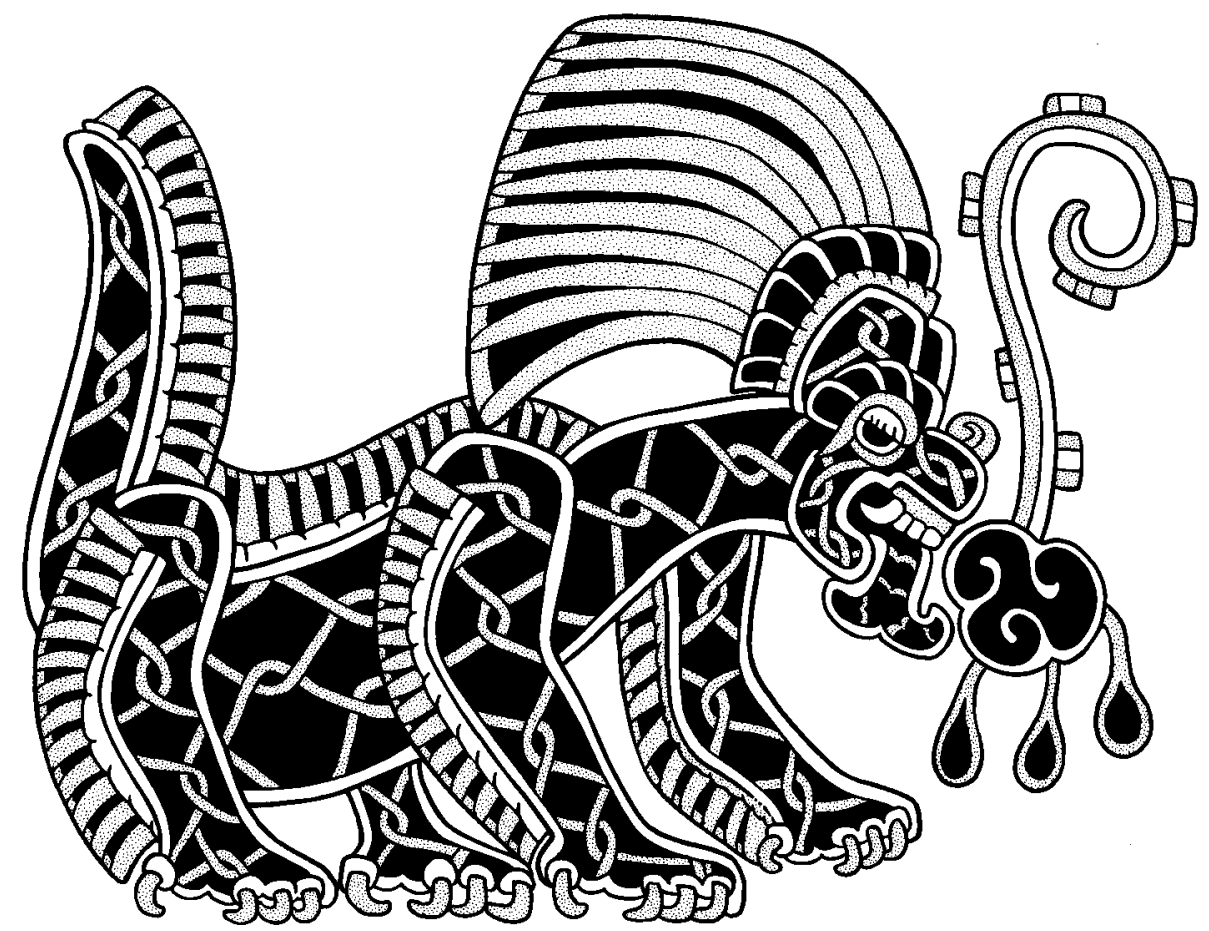

FIG. 7 - Félin réticulé à la coiffe, rehaussé de plumes et laissant apparaître une langue bifide (Atetelco) [dessin N. Latsanopoulos].

tant que forme générale du papillon et celui-là en tant que motif. Reste le problème de la langue bifide qui ne cadre pas avec l'ensemble de ces compositions.

Ainsi, sur la série d'appliques ou d'éléments en forme de papillon que l'on voit sur les encensoirs ou sur des sceaux de cette tradition, le bas des abdomens des lépidoptères est traité de façon à faire apparaître deux éléments divergents, qui prennent la forme d'une sorte d'ancre marine arrondie. Or cet élément iconographique a été reconnu par Beutelspacher comme reprenant une caractéristique anatomique de l'appareil reproducteur des mâles de papillons : les valves se trouvent placées à l'extrémité inférieure de l'abdomen et s'ouvrent en une structure symétrique bifoliée. Les artistes teotihuacan ont ainsi rendu un élément visible de l'anatomie des lépidoptères sous les traits d'une «langue » bifide (Figure 8). 

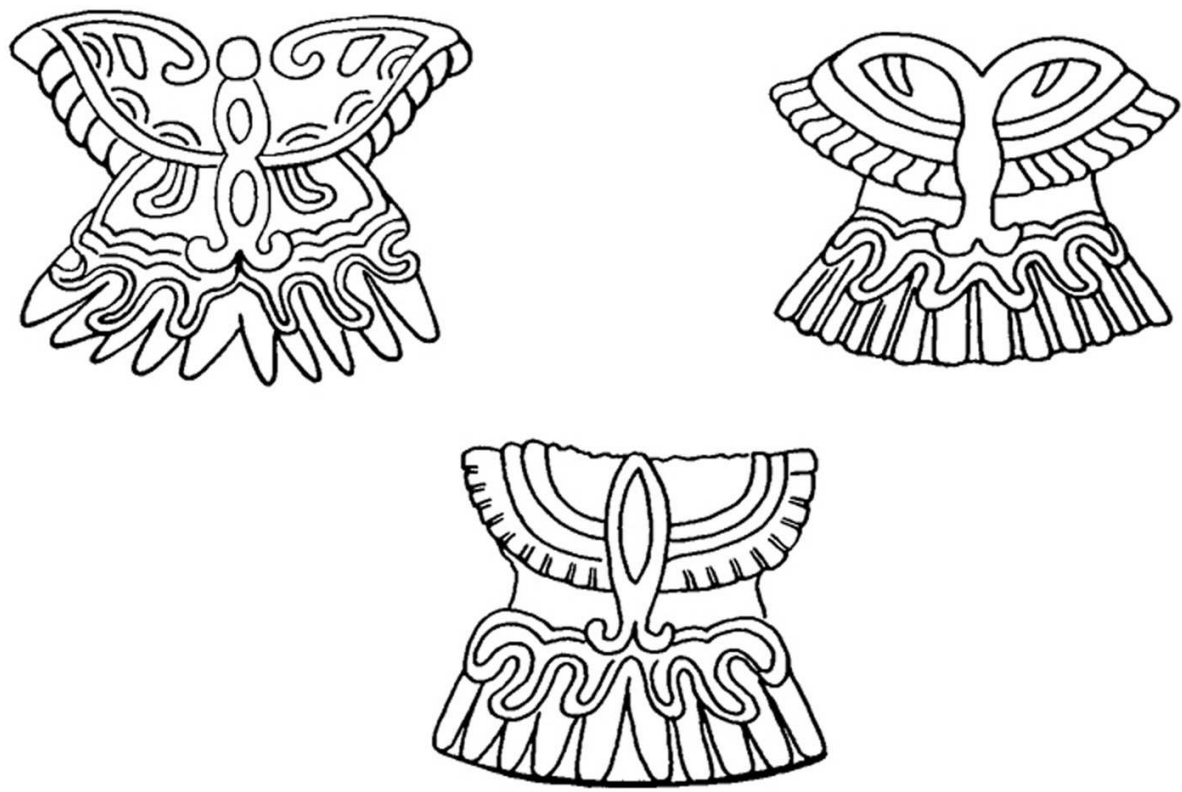

FIG. 8 - Appliques de Teotihuacan en forme de papillon laissant apparaître l'appareil reproducteur mâle présent à l'extrémité inférieure de l'abdomen qui sera repris en tant que langue bifide dans les compositions mêlant papillon et motif de face de jaguar [d'après Van Dinter 2006, p. 187].

Une fois placée en association avec le motif de la gueule ouverte d'un félin donné par les dessins des ailes du papillon, cette extrémité bilobée de l'abdomen évoque, chez un observateur extérieur, l'image d'une langue bifide. Cette langue ne peut être celle d'un jaguar, ni d'un félidé, ni d'ailleurs d'un quelconque mammifère, la langue des mammifères n'apparaissant jamais sous cette forme, contrairement à certains reptiliens, notamment les serpents. En réalité, c'est donc l'image générale d'un papillon qui peut être retranscrite comme « tête de félin à langue de serpent $\gg$.

Encore une fois, la combinaison coiffe-papillon/tête de jaguar à langue de serpent n'est que le résultat d'une description, en des termes imagés, d'un ensemble de caractéristiques présentes sur un seul être, le papillon. Leur réunion dans l'art de Teotihuacan n'est donc pas le résultat d'une volonté culturelle de présenter les trois règnes réunis au sein d'un seul être hybride par l'intermédiaire de leurs représentants emblématiques, mais plutôt celui d'une description d'une forme existante, certes énigmatique, mais non moins perceptible matériellement. Toutefois, ce constat n'épuise pas la figure du jaguar réticulé sur laquelle nous reviendrons dans une autre contribution. 
La même analyse doit maintenant être menée pour le serpent à plumes, une des figures composites majeures du répertoire iconographique de Teotihuacan.

\section{LES « SERPENTS À PLUMES » ET LES « SERPENTS DE FEU »}

Les dénominations « serpent à plumes » et « serpent de feu » viennent, d'un côté, de la désignation d'une figure centrale de la religion de Teotihuacan, Quetzalcóatl et, de l'autre, d'une figure importante de la pensée aztèque, les Xiuhcóatl. Littéralement toutefois, "Quetzalcóatl» signifie serpent (coatl)/ oiseau quetzal (quetzal). La polysémie des termes recouvre plusieurs possibilités, mais le fait d'avoir opté pour la désignation de « serpent à plumes » est a priori essentiellement dû à la forme donnée à cette entité dans l'iconographie. Comme nous l'avons vu avec les poissons plus haut, en Mésoamérique le passage par l'iconographie implique de prendre en considération plusieurs facettes des modalités de la figuration. Ainsi, un « poisson-colibri », même s'il est figuré avec un bec de colibri, n'est pas un être imaginaire mi-poisson, mi-colibri : il est juste un poisson dont le « bec » (la bouche), rappelle, par sa forme, celle de l'oiseaumouche. Bien que sa figuration semble naturaliste, elle n'est qu'une mise en image et se rapproche d'un logogramme ou d'un « icônogramme ».

Linguistiquement parlant, le terme «Quetzalcóatl » est construit de façon analogue aux termes désignant les poissons (totomichin, etc.). Il faudrait donc, en premier lieu, s'interroger sur l'existence d'un être qui puisse être désigné par les images qu'il renvoie, avant d'entériner la nature imaginaire et monstrueuse qu'il adopte dans l'iconographie. De fait, le terme de coatl peut être utilisé pour désigner d'autres classes d'êtres que des reptiles. Ainsi, les vers intestinaux tzoncoatl $^{5}$ ne sont pas des serpents au sens strict du terme, même s'ils en possèdent en gros la forme, raison pour laquelle ils peuvent être désignés de la sorte. Sahagún (1950-1981) ${ }^{6}$ fait même remarquer qu'en nahuatl classique, le chemin pouvait se dire coatl, un substantif, comme nous utiliserions le terme de « serpent » en tant que prédicat pour dire d'un sentier qu'il « serpente » entre les collines ou dans les bois. C'est bien l'analogie formelle qui permet cette désignation, bien qu'il nous soit moins familier d'utiliser une forme substantivée pour exprimer la même idée, puisqu'une phrase comme celle-ci : « il faut emprunter ce serpent pour parcourir les collines » ne convient pas en français, sauf peut-être sous l'angle de la licence poétique. Il est donc licite, en nahuatl pour le moins, d'aller chercher le terme qui signifie un être ou une chose pour désigner une partie d'un tout qui en possèderait la forme. Le terme coatl sert ainsi à nommer, en plus des serpents, ce qui a la forme d'un serpent, et non exclusivement les ophidiens.

Plusieurs chenilles de différentes espèces de papillons porte-queue - de la même famille que ceux que l'art teotihuacan a choisi de représenter à profusion sur ses encensoirs - ont une forme mimétique propre qui les fait gonfler et 
redresser leur thorax lorsqu'elles se sentent menacées et ressembler à des serpents, cela afin d'éloigner les prédateurs. Ces formes mimétiques laissent apparaître de faux yeux sur les segments du thorax de la chenille et ce processus a même produit un organe invaginé au dessus de la tête - évaginé lorsqu'il est apparent qu'aucune autre famille de papillon ne possède, l'osmeterium, à l'odeur nauséabonde pour les prédateurs et qui, avec l'aspect général de serpent, en vient à former très exactement l'image de la langue bifide d'un ophidien (Figure 9). Ainsi, Beutelspacher transcrit les informations suivantes d'après la chronique de Fernández de Oviedo y Valdés écrite au Xvi siècle $^{7}$ : "Se encuentra entre los mechoacanes otra especie de gusano llamado en su lengua Itzugua y Sipantipe, con dos cuernecillos rojos con que tantea su camino; su figura es monstruosa y su cuerpo de color negro y pardo. Quise agregarlo a los demás, porque huele exactamente igual que los melones (C. X) » (Fernández de Oviedo y Valdés in Beutelspacher 1988, p. 22). Beutelspacher précise qu'il s'agit sans aucun doute des chenilles de la famille Papilionidae et ajoute qu'il ne comprend pas le sens des deux termes purépecha. Placée en haut de la tête de la chenille et rétractile, la «langue de serpent » forme une sorte de corne unique qui se divise en deux à son apex.

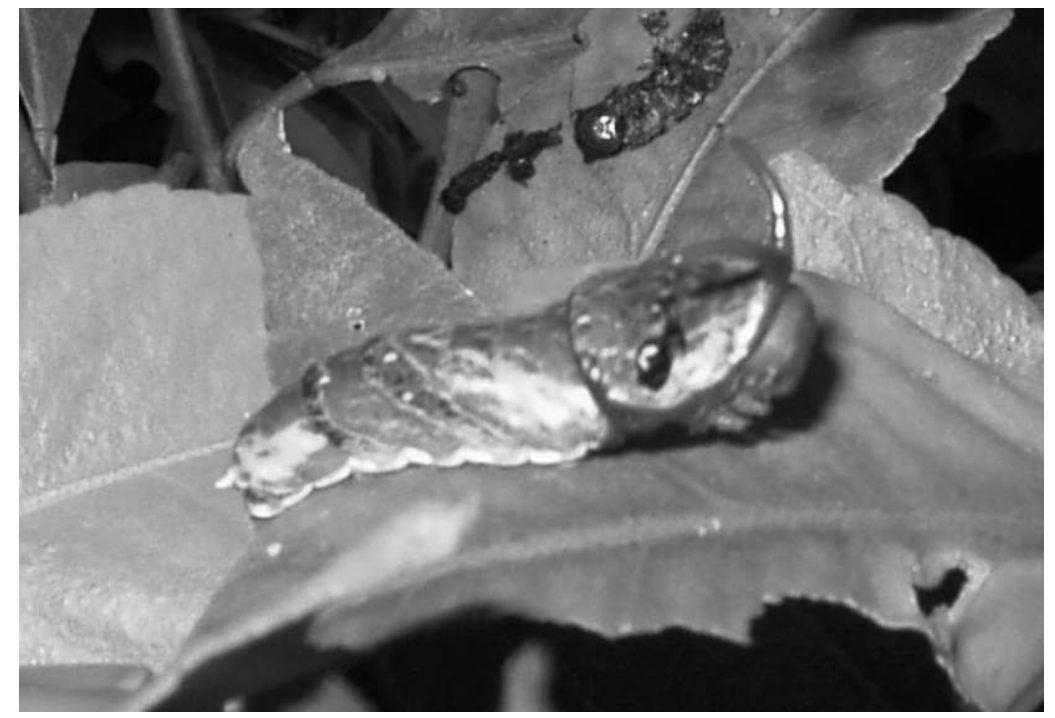

Fig. 9 - Chenille de Papilio multicaudatus à la forme mimétique d'un serpent avec une structure évaginée orange formant l'image d'une langue de serpent [cliché de l'auteur D. K.].

Ce type de chenilles peut recevoir l'appellation du serpent puisqu'il en a l'aspect, comme le souligne Beutelspacher. Parmi certains noms de chenilles, Molina indique, dans son vocabulaire nahuatl de [1571] 1966: " Ocuilin cuacuae, gusano con cuernecillos. También dice mazacouatl ». "Y probablemente se refiere, 
por los cuernecillos, al osmaterium ", ajoute Beutelspacher (1988, p. 21). En nahuatl, cuacuahuitl désigne les cornes et les antennes des insectes, alors que le terme général pour les chenilles et certains vers est ocuilin : ocuilin cuacuae est donc une désignation littérale d'une "chenille à cornes». Le second terme, maza-coatl, fait référence à ce que cette même chenille évoque : coatl est alors utilisé pour désigner son aspect, celui du serpent, alors que ses «cornes » sont rendues par l'apocope d'un terme, mazatl, qui désigne le cerf, ce qui en fait, sous cet aspect, un « serpent-cerf ». En ce sens, l'osmeterium forme, pour les locuteurs nahuatl, une « corne » de la chenille, bien que son aspect soit celui d'une langue de serpent (transcrit par le terme de coatl, lequel s'explique aussi par l'allure générale de la larve). Selon Sahagún (1950-1981, XI, p. 166), mazacoatl sert aussi à désigner trois serpents, parmi lesquels un de la famille des crotalidés, Crotalus cerastes, ou « crotale à cornes ». Les rapprochements qui ont, jusqu'ici, été faits de la présence de «cornes» dans les images des serpents monstrueux, comme ceux de Teotihuacan ou des Aztèques, renvoient à ces espèces de serpents, mais laissent de côté la référence à la chenille. La présence de la structure vibrante du bruiteur du crotale dans les iconographies explique certainement la reconnaissance des crotales, mais laisse de côté l'association possible avec la chenille qui, à notre avis, ne devrait pas être évacuée aussi rapidement ${ }^{8}$.

Le terme de coatl intervient également dans le nom qui sert à désigner une autre chenille : « Según Robelo, llamaron coguapochi (de cohuapochin, de cóatl, culebra, y pochin, apócope de pochinque, cardado, "culebra cardada") a una especie de gusano del grueso de un dedo, cuya picadura es peligrosa. Los pelos blancos que cubren su cuerpo parecen algodón cardado, de donde vino el nombre » : il s'agirait, selon Beutelspacher (1988, p. 21), des chenilles velues des Mégalopyges ${ }^{9}$. Le terme coatl serait cette fois-ci employé en raison de l'aspect urticant des poils de la chenille puisque leur contact est comparable à l'injection d'un venin, autrement dit, le danger que l'animal représente le ferait classer, par métonymie, comme un serpent. Coatl pourrait ainsi servir à désigner des chenilles sous les deux modalités de l'aspect et du comportement du serpent ou de l'effet induit par sa morsure.

Étant donnée l'importance centrale des papillons dans l'univers des représentations mésoaméricaines, les données présentées ci-dessus devraient permettre de repenser ces figures centrales que sont les serpents à plumes et les Xiuhcóatl. À la suite de Darlington qui, en 1931, proposa que les « serpents de feu » auraient eu pour origine une retranscription imagée de la chenille, Taube propose une interprétation analogue pour les Xiuhcóatl lorsqu'il affirme : " It will be subsequently noted that like the War Serpent, the Xiuhcóatl also represents a caterpillarlike being " (2000, p. 285), et l'on se réfèrera à la comparaison qu'il fait entre les iconographies mexicaines représentant des chenilles et celles de Xiuhcóatl (ibid., fig. 10.12), puisque, selon lui, " [...] along with their long, tubular bodies, caterpillars also possess legs, which are particularly developed in the frontal thoracic 
region, recalling the prominent forem-limbs of many Teotihuacan War Serpents » (ibid). Taube, pourtant, ne note pas que c'est une qualité possédée par la chenille même, son aspect extérieur issu de sa faculté mimétique, qui fait de l'un - l'aspect serpent - le déguisement de l'autre - la chenille. Enfin et surtout, il ne fait pas cette association entre chenille et Quetzalcóatl dans sa publication de 2002, puisqu'il considère que, contrairement aux autres serpents fantastiques, l'aspect de ce dernier emprunte directement sa forme à celle des serpents. Nous pensons au contraire qu'il est possible de montrer que les serpents à plumes de quetzal sont également une transcription imagée d'une famille de chenille.

\section{LES SERPENTS FANTASTIQUES COMME IMAGES DE LA CHENILLE}

L'aspect général des chenilles en forme de serpent devient ainsi problématique au regard de l'iconographie mésoaméricaine. En effet, si deux êtres apparaissent imbriqués l'un dans l'autre et, bien que l'un des deux ne soit qu'une image, cette combinaison pose un problème de définition des êtres à un système de pensée comme le nahualisme qui considère que l'aspect (ou la physicalité, pour reprendre une distinction descolienne) est tout aussi important que l'être (ou l'intériorité). Renvoyer l'aspect d'un serpent, c'est l'être en partie. Dans le sens de double, le nahualli de cette chenille pourrait ainsi être le serpent ; il en est le « déguisement ». Le point qui nous importe, dans un premier temps, est qu'un élément unique de l'anatomie d'un être puisse, en nahuatl, recevoir plusieurs termes pour le désigner. Dans un second temps, il faut souligner que le registre dans lequel sont puisés ces termes relève le plus souvent de la simple description, mais aussi tient à l'image que cet être renvoie, au même titre que le font les différents « poissons-papillon » et « poissons-jaguar » du Códice Florentino ( $c f$. Figure 1).

Revenons à la description d'une chenille de la famille des Papilio suivant deux registres, celui de sa qualité de chenille et celui de sa modalité mimétique (" serpent »). La combinaison de ces deux registres au sein d'un être unique fait que chacune des images mimétiques portée par la chenille peut être combinée à la place qu'elle occupe au sein de l'être réel et former, de la sorte, un être hybride. Ainsi, la tête de la chenille prend la place du museau ou du « nez » du serpent tout en étant à l'intérieur de sa " gueule », alors que les mandibules jouent le rôle des crochets ou dents de devant du « serpent » (cf. Figure 10 et I haut dans le cahier en couleurs et Figure 11). Dans la Figure 11a, on voit que les yeux des serpents de feu sont une retranscription imagée des faux yeux, ou ocelles, que la chenille porte en tant que livrée mimétique sur son thorax. Ensuite, la division de la tête de la chenille en deux parties circulaires les fait apparaître chacune comme un œil pour la chenille. De fait, les « cornes » enroulées, que forme l'osmeterium, lequel, nous l'avons vu, renvoie à l'image de la langue du serpent, se trouvent placées en haut de ces « yeux ». Cette correspondance est ainsi à même d'expliquer la présence de 
« cornes », enroulées de surcroît, dans la continuité des «yeux » des serpents monstrueux dans les images de Teotihuacan, comme celles du serpent à plumes. Enfin, chaque tête de chenille possède six vrais yeux, des stemmates ou œil simple des formes larvaires des insectes : ensemble, ils forment un amas ou suivent le tracé d'une sorte de point d'interrogation ( $c f$. Figures $11 \mathrm{~b}$ et c). Lorsqu'est considérée l'image mimétique de la chenille, ces stemmates sont placés au bout du museau du « serpent » et constituent la corne enroulée ornée d'yeux des serpents de feu ( $c f$. Figure 11a). Les trois paires de vraies pattes de la chenille forment, quant à elles, l'image des dents arrière du serpent, alors que le reste de son corps est divisé en segments.

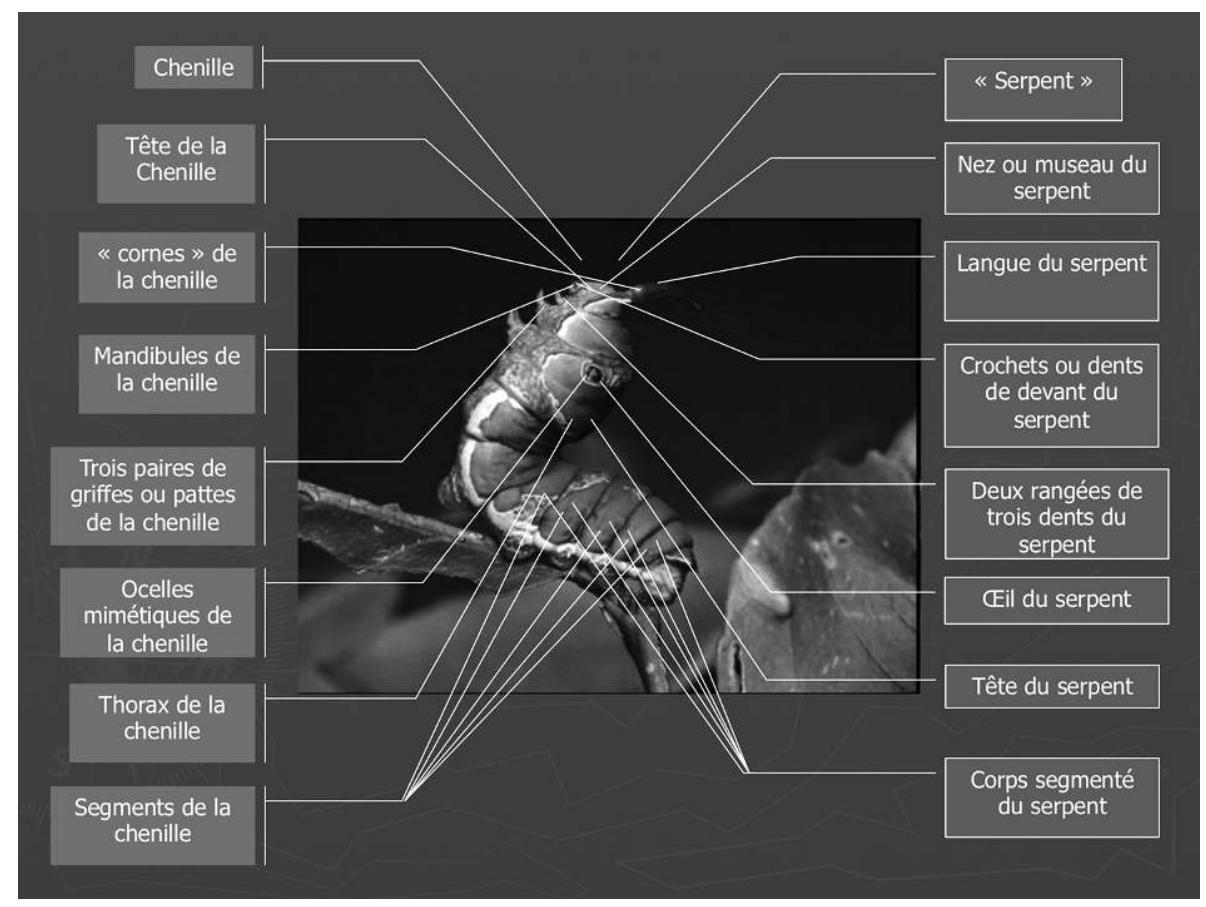

FIG. 10 et I haut dans le cahier en couleurs - Description des parties constitutives des chenilles de Papilio sp. suivant deux registres : celui de son anatomie et celui de son aspect mimétique de serpent [photocomposition de l'auteur, D. K.].

Cet ensemble de caractéristiques est partagé par les Xiuhcóatl, ou « serpents de turquoise » présents sur la pierre du calendrier solaire où les deux entités solaire et terrestre - Tonatiuh et Xiuhtecutli « dieu de turquoise » - apparaissent dans la gueule des deux « serpents de feu » (Figure 11a). Chacun est doté d'une corne recourbée qui part du museau et sur laquelle apparaissent sept yeux 
- parfois huit dans les illustrations des codex - qui seraient une évocation de la constellation des Pléiades, suivant que sept ou huit étoiles qui constituent cet amas ouvert dans le ciel étoilé sont prises en compte. Dans la continuité de Darlington (1931), Taube a admis en 2000 que les Xiuhcóatl étaient une transcription imagée de chenilles, sans toutefois proposer d'espèces spécifiques, ni souligner l'aspect mimétique de certaines d'entre elles.

Or la disposition des yeux sur la « corne » des serpents fantastiques suit celle des stemmates sur la tête de la chenille où ils prennent la forme d'un point d'interrogation. Ils sont placés selon le plan anatomique du « serpent », c'est-àdire sur son " museau », là où sortirait la « langue » du monstre ophidien. Nous l'avons vu, cette langue est présente sous la forme de l'osmeterium, mais représente une corne pour la chenille. En cela, les cornes dotées de sept yeux ou étoiles devraient ainsi être considérées comme la retranscription graphique des stemmates des chenilles sous leur forme fantastique. Enfin, les têtes des déités qui apparaissent dans la gueule des monstres sont celles des chenilles.

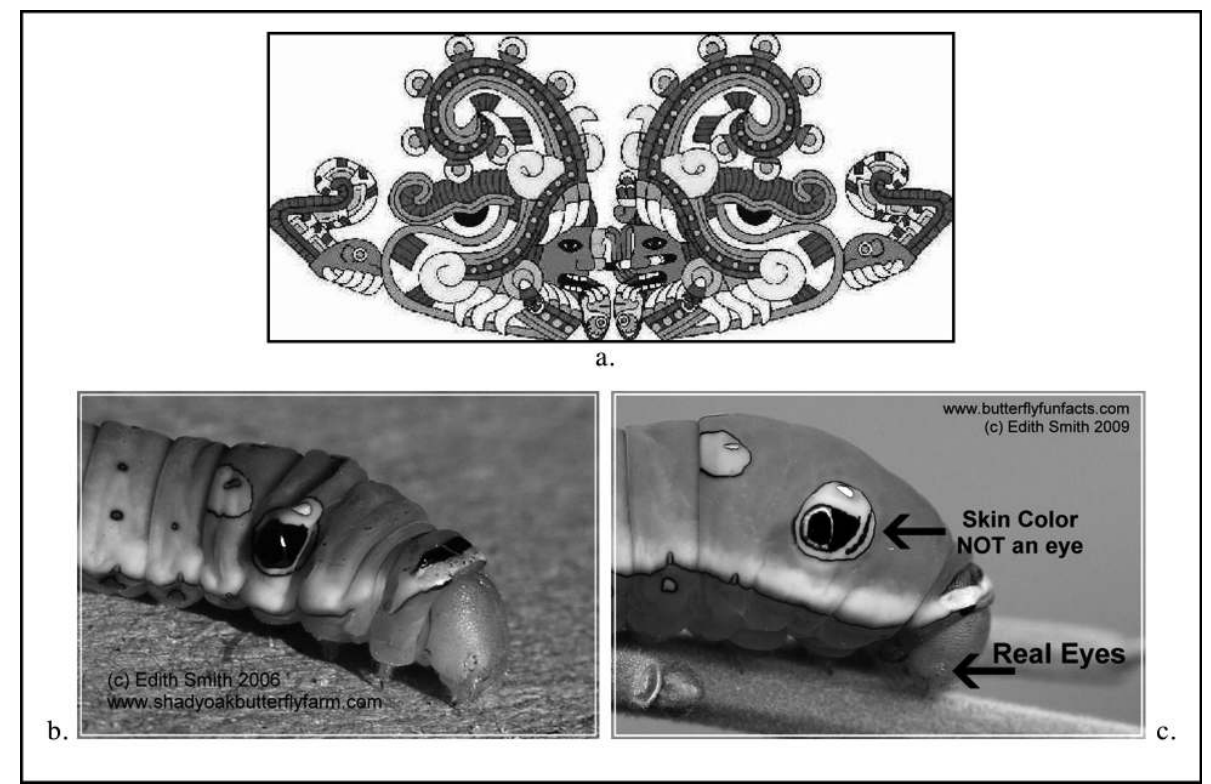

FIG. 11a, b et c - Tonatiuh et Xiuhtecutli sortant de la gueule des serpents de feu sur la « Pierre du soleil ». Chacune des «cornes» des serpents fantastiques est le support de sept yeux qui pourraient correspondre aux stemmates - ou vrais yeux-présents sur la tête des chenilles [Fig. 11a: d'après http://www.samaelgnosis.net/calendario_azteca/cap05_binario_ serpentino.html ; Fig. 11b : d'après www.shadyoakbutterflyfarm.com ; Fig. 11c : d'après www.butterflyfunfacts.com]. 


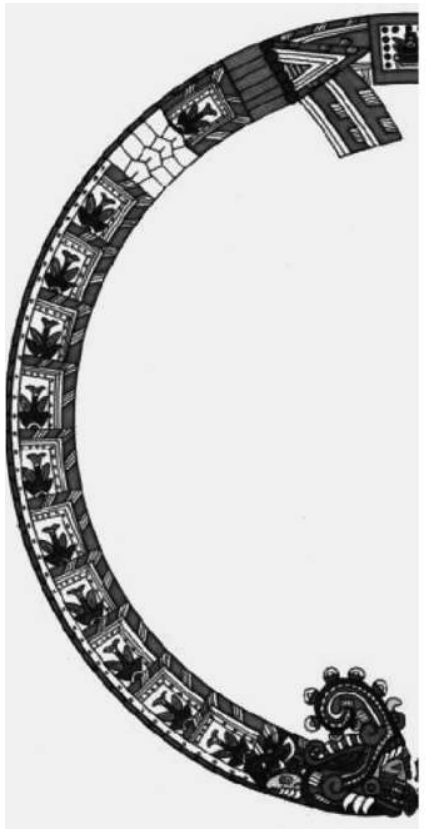

a.

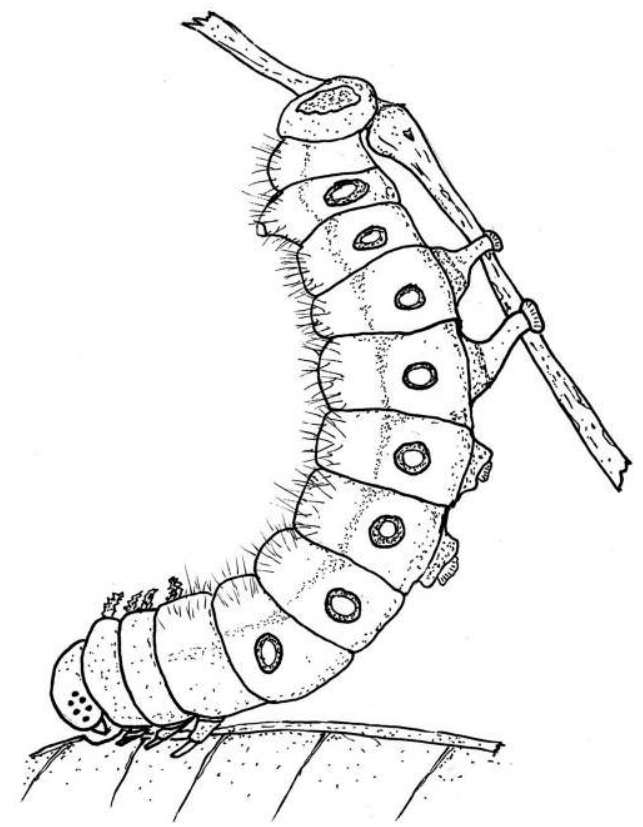

b.

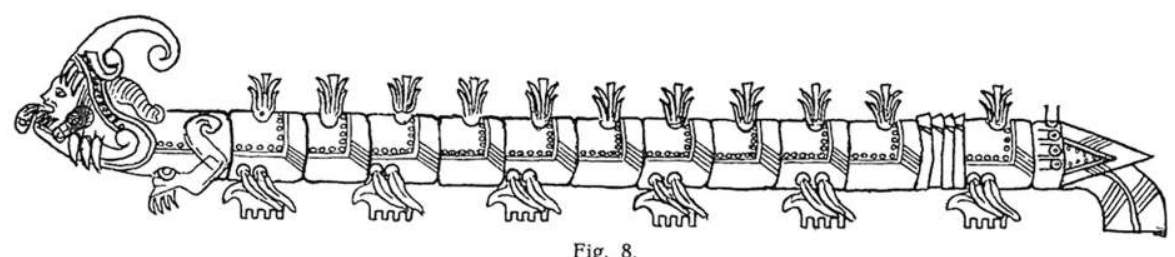

Fig. 8.

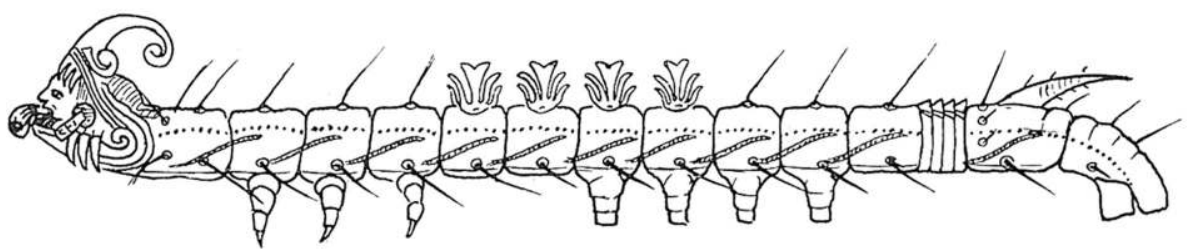

Fig. 9.

c.

FIG. 12a, b et c - Mise en parallèle de la structure d'un Xiuhcóatl avec celle d'une chenille de façon analogue à celle proposée par Darlington [Fig. 12a : d'après http://www.samaelgnosis.net/ calendario_azteca/cap05_binario_serpentino.html.; Fig. 12b : dessin de l'auteur D.K. ; Fig. 12c : Darlington 1931, figs. 8 et 9]. 
Par ailleurs, les Xiuhcóatl possèdent treize segmentations en référence à la « treizaine » du calendrier aztèque. Or, comme Darlington le remarquait déjà (1931, p. 640), les chenilles ont une segmentation analogue, c'est-à-dire que leur corps est constitué de treize segments, trois sur le thorax et dix formant le reste du corps, sans compter la tête (Figures 12a et b). Sur la chenille, chacun des segments, hormis deux des trois du thorax, possède un stigmate apparent sous forme de petites ouvertures à partir desquelles s'organise la livrée de la chenille. Ces stigmates apparaissent en tant que motifs sur les segments des Xiuhcóatl. Sur les serpents de feu, le caractère « brûlant » est souligné par une série de motifs de papillons stylisés placés là pour évoquer l'image de flammes ou de flammèches (Figure 12c).

$\mathrm{Au}$ même titre que les glyphes, les figures présentes sur les monuments devaient ainsi être perçues par les Indiens comme des combinaisons de termes figurés par les images des constituants de leur nom. Dans cette logique, tout coatl n'est donc pas un serpent puisqu'il peut renvoyer à une chenille. Dans le cas du serpent en Mésoamérique, l'utilisation de l'image relève autant de la figuration que de la nomination. Notre propre tradition ayant tendance à appliquer aux images une grille de lecture figurative, nous ne pouvons que faire entrer ces êtres hybrides dans la catégorie des " êtres surnaturels », puisqu'ils ne figurent rien d'existant à nos yeux, dans la mesure où la grille ontologique naturaliste conditionne notre regard. Par conséquent, la façon dont nous, observateurs extérieurs d'une culture donnée, avons tendance à percevoir les productions iconiques conditionne l'imputation de croyances spécifiques aux producteurs de ces images. Il faut encore ajouter à cela que notre forme d'écriture phonétique dissocie graphie et figuration au contraire de la modalité graphique mésoaméricaine qui maintient dans ses glyphes une dimension figurative. La conséquence inverse de ce constat est que toute figuration est également, dans ce système, une invitation à lire des formes.

\section{Quetzalcóatl ou la CHenille À Plumes de QueTzal}

Avant d'évoquer la polysémie du terme « quetzal », il faut examiner quelques emplois de la plume dans l'iconographie de Teotihuacan, celles des poteries zapotèques et des codex aztèques. Les coiffes de la céramique zapotèque, qui intégraient des représentations de papillons par exemple, étaient constituées de plumes pour former aussi bien l'image des ailes de lépidoptères qu'orner leurs antennes et leur trompe. Même si, chez les Aztèques, les habitants de Teotihuacan et les Zapotèques, les coiffes ne sont pas des artéfacts exclusivement ornés de plumes, puisque de la peau, des os, des coquillages, du papier, des pierres et d'autres matériaux y interviennent, c'est pourtant la plume qui entre le plus communément dans leur confection. De la sorte, lorsque, dans la langue nahuatl, le qualificatif anecuyōtl « coiffe » (ici celle de Huitzilopochtli) désigne 
des éléments portés par d'autres êtres, à la manière qu'ont les humains d'utiliser les phanères des oiseaux pour s'en confectionner des parures, c'est à l'image de la plume que ces éléments renvoient. Pour un être qui porte des excroissances ayant la forme de houppes ou d'antennes, c'est une relation anthropocentrée qui fait choisir le terme en référence aux plumes portées par des danseurs ou des guerriers. En ce sens, dire « coiffe » ou « parure » en nahuatl, c'est évoquer la plume. On utilisait ainsi la plumasserie pour figurer ces éléments non humains dans les rituels ou dans les costumes. Les quetzalcuacuahuitl sont deux plumeaux portés en panache sur le haut de la tête de danseuses à la façon de toupets dans le costume de Xochiquetzal : le terme est formé sur celui qui désigne les cornes et les antennes des insectes (cuacuahuil) auquel le terme quetzal est accolé dans son sens de plume (omequetzalli). Xochiquetzal, déesse de la beauté, des arts et de la sexualité, était parfois présentée sous des traits de papillons de la famille des Porte-queue ( $c f$. Beutelspacher 1988). Lorsque, dans les figurations des codex par exemple, les artistes aztèques décident de lui donner les traits d'un papillon, ils figurent parfois ses antennes par des plumes de quetzal, plutôt que de prendre le parti d'en faire une figuration naturaliste que rien ne leur interdirait d'entreprendre. Dans ces traditions, une composition picturale qui apposerait des plumes à un être ne signifie pas que l'être en question en soit réellement doté, à l'instar des antennes du papillon figurant Xochiquetzal. Plus prosaïquement, l'être en question porte des éléments qui évoquent l'image de la plume. En tant qu'artéfact, la plume peut ainsi signifier autre chose qu'un élément aviaire, comme sur les coiffes en forme de papillon. Il faut également considérer l'équivalent inverse de cette proposition : si certaines excroissances peuvent renvoyer à l'image de plumes, c'est cette dernière image qui sera employée dans les compositions iconographiques plutôt qu'une figuration naturaliste de l'élémentsource ${ }^{10}$.

Dans les figurations de Quetzalcóatl, c'est l'aspect « serpent à plumes » qui prime, et cette combinaison a également été retenue par certaines interprétations pour souligner qu'une matière associée à l'élément aérien, la plume, est combinée à un être chtonien, le serpent, dans le but d'exprimer la réunion de deux aspects irréconciliables ( $c f$. Séjourné 1962, p. 35). Chez Nicholson (2000, p. 145), on lit également "This union of the precious green feathers of a bird, symbolizing the celestial realm, and a dangerous, slithering reptile, connoting the terrestrial sphere, this fusion of earth and sky - as in many cosmologies - signified, above all, fertility and creativity." "Or les constatations que nous venons de faire, tant en ce qui concerne le terme de coatl que la plume, laissent entrevoir que la combinaison « serpent à plumes » n'évoque pas nécessairement un ophidien emplumé, mais plutôt, sous sa compréhension figurée, un être à l'aspect de serpent doté d'éléments portés sur son corps et décrits comme des plumes. Remarquons encore que, si le terme de «Quetzalcóatl » est formé sur celui de serpent, celui de quetzal ne renvoie pas uniquement à l'oiseau ou à ses plumes, mais sert aussi à marquer une 
couleur, le vert profond. C'est ce que fait remarquer Thouvenot dans son ouvrage sur le jade en Mésoamérique indienne (1982) pour le quetzalchalchihuitl, une forme de jade hautement valorisée pour sa caractéristique chromatique. Il faut donc associer les deux formes, l'évocation de la couleur et celle de la plume, pour comprendre quel type de coatl est ainsi désigné.

Les figurations de serpents à plumes sur le temple de Teotihuacan portant le même nom et sur les fresques murales de cette tradition laissent apparaître un être composite dont les éléments constitutifs peuvent être reliés à des formes visuelles d'un être réel qui les possèdent pour la plupart. Une composition iconique et analogique, en quelque sorte, issue d'une description d'un être réel. Sur les figurations teotihuacan du serpent à plumes qui, pour Sugiyama (2000), sont à l'origine des autres formes mésoaméricaines, et bien que cela n'apparaisse que sur le serpent à plumes de la peinture murale de Techinantitla, les plumes sont placées à intervalles réguliers sur une série de segments séparés chacun par de minces bandes jaunes. Or les serpents n'ont pas de corps segmenté : il s'agirait donc plutôt de la figuration d'une segmentation qui existe chez d'autres espèces. Ce corps segmenté de serpent à plumes n'est pas sans évoquer celui des chenilles, comme nous l'avons vu pour les Xiuhcóatl. Le terme «Quetzalcóatl » peut ainsi désigner un « serpent à plumes », mais aussi une « chenille à plume de quetzal », au même titre que mazacoatl, « serpent-cerf » est une « chenille à corne de cerf », puisqu'il n'est pas nécessaire de dire d'un poisson-chat qu'il est un « poisson aux vibrisses de chat », ou d'un « poisson-perroquet » qu'il est un « poisson au bec de perroquet » : la saillance visuelle qui fait pont est oblitérée au profit de la simple apposition des noms des deux espèces. Il n'est donc pas nécessaire de dire un «serpent aux plumes de quetzal», mais « serpent-quetzal», sous-entendu « chenille-quetzal » (Figure 13).

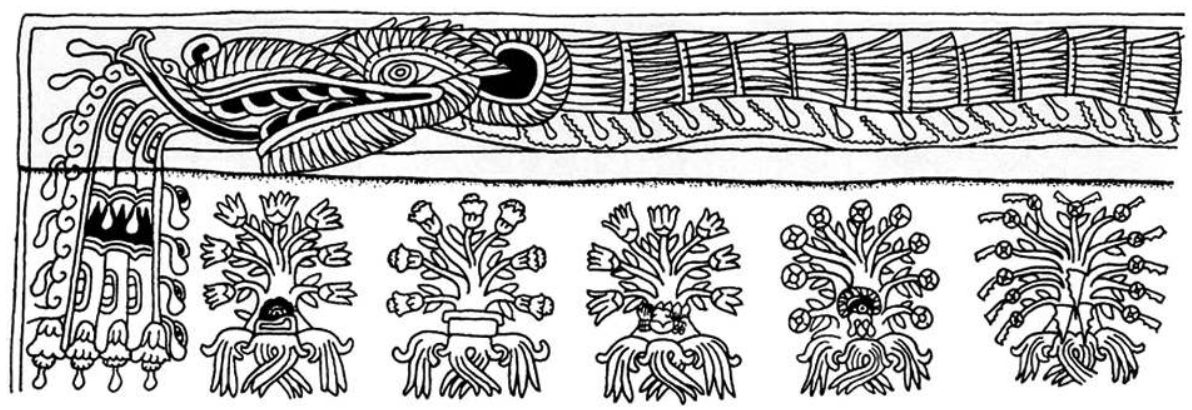

FIG. 13 - Figuration du « serpent à plumes » dans l'art teotihuacan (peinture murale de l'ensemble résidentiel de Techinantitla). On notera le corps segmenté ainsi que la répartition des plumes disposées sous forme de rangées analogues [d'après Taube 2002 ; voir aussi Berrin 1988]. 
Puisque certaines chenilles renvoient l'image du serpent, il est légitime de les qualifier de la sorte. Or il existe un ensemble plutôt large de chenilles qui portent une profusion de poils, urticants pour certains, qui forment chez plusieurs espèces de véritables houppes multicolores et dont la répartition symétrique et harmonieuse sur l'ensemble du corps n'est pas dénuée d'un effet esthétique impactant pour un observateur humain. Ainsi, Fernández de Oviedo y Valdés fait une description d'une chenille à poils urticants dont la structure empennée ressemble, par la forme, à celle de l'asperge sauvage, et dont la couleur varie du blanc au vert irisé en passant par le jaune, chenille à la tête bleue turquoise et qu'une ligne jaune traverse de tout son long :

Del Coahuapochin. Es un gusanillo ceniciento, peludo, cubierto de unas como ramillas verdes con hojitas muy delicadas (por decirlo de alguna manera) algo parecidas a las del espárrago silvestre, dispuestas en hileras y a intervalos sobre el dorso, el cual está cruzado de rayas negras transversales, en el mismo sentido de las ramillas; rematan éstas a uno y otro (lados) en una línea amarilla que se prolonga rectamente. Es más o menos del tamaño de un dedo. La cabeza, las patas y la cola son de color blanco, azul y escarlata bellamente combinados. Su mordedura es nociva y produce un fuerte ardor en las partes mordidas. Hay otros innumerables géneros de gusanos muy hermosos, que fácilmente pueden conocerse por lo que hemos pintados como muestras (Fernández de Oviedo y Valdés in Beutlespacher 1988, p. 22) [Les dessins ont, hélas, été perdus.]

Beutelspacher n'identifie pas exactement cette chenille et suggère qu'elle appartiendrait à la famille des Saturnidés. Pourtant, la description de Fernández de Oviedo y Valdés semble faire référence à la chenille des Automeris, à tête bleue, dont les poils urticants forment des structures empennées vertes qui ne sont pas sans rappeler des plumes, et plus particulièrement celles du quetzal. Ainsi, les rectrices de l'oiseau quetzal possèdent une structure analogue à la branche de l'asperge sauvage (Asparagus acutifolius); leur partie terminale dissocie les barbules, ces éléments qui forment la penne de la plume et les laissent apparaître comme des feuilles de cette plante. Cette formation en " touffes ", à la manière de l'asperge sauvage, est caractéristique des épines des chenilles d'Automeris et se retrouve aussi sur des figurations de "serpents à plumes » de périodes plus tardives que Teotihuacan, comme sur un sceau au serpent «monstrueux » (cf. Figure 14).

Le nom nahuatl donné à cette chenille Coahuapochin est en fait le même que celui donné par Robelo à une autre chenille urticante de la famille des Mégalopyges. Le terme signifie « serpent-cotonneux », par référence aux poils blancs de la chenille ( $c f$. supra). Cela dit, la description donnée par Fernández de Oviedo y Valdés ne correspond pas aux chenilles de cette famille des Mégalopyges, mais plutôt à celle du genre Automeris et Lonomia de la famille des Saturnidés. Or Sahagún décrit par le même nom de Auatecolotl, qui fait référence au hibou, une autre espèce de chenille aux propriétés urticantes : " Ay otros brugos que llaman 


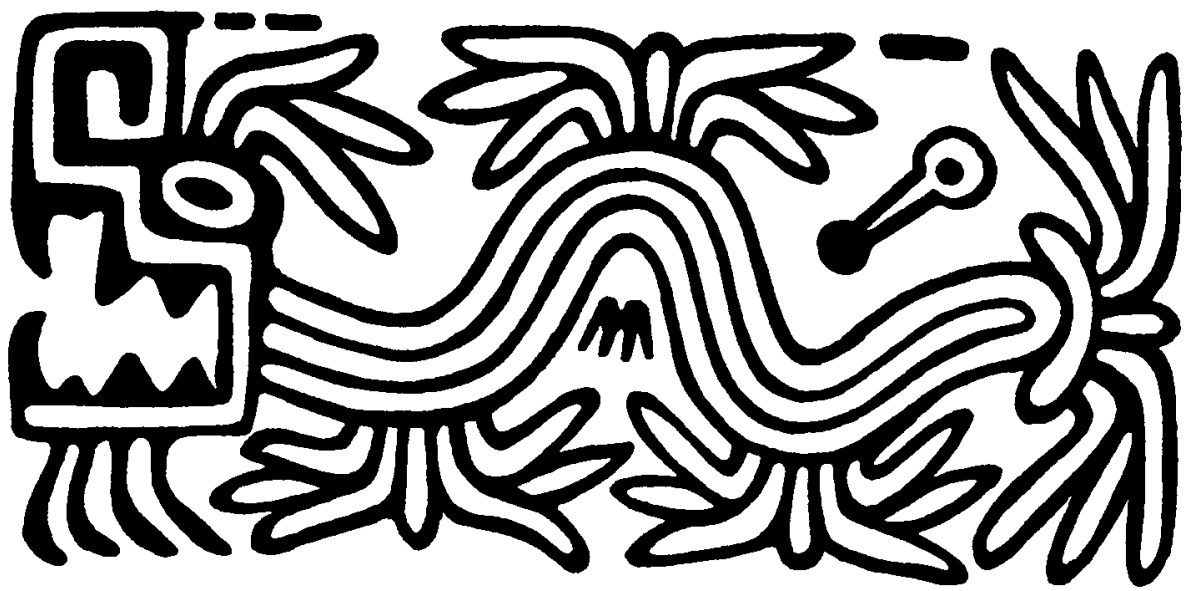

FIG. 14 - Serpent à plumes sur un sceau de période mexica. La répartition des plumes se fait en plumeaux ou en touffes [d'après Enciso 1947].

Auatecolotl: tambien se crian en los arboles. Unos son negros, otros rosos, son muy bellosos, y los pelos que tienen pican. Las picaduras doelen como picadura de alacran: tambien se vuelven mariposas » (Sahagún 1950-1981, XI, f. 103r ; Figure 15).

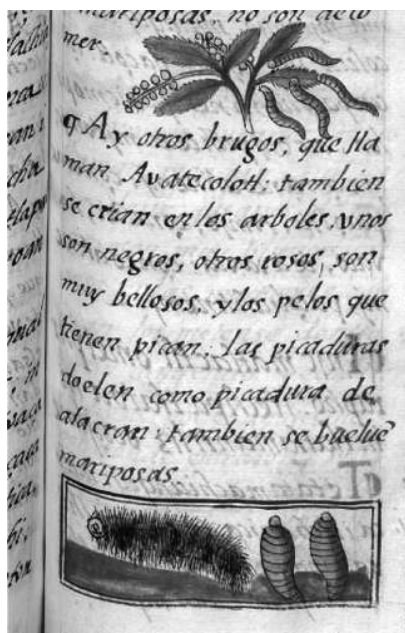

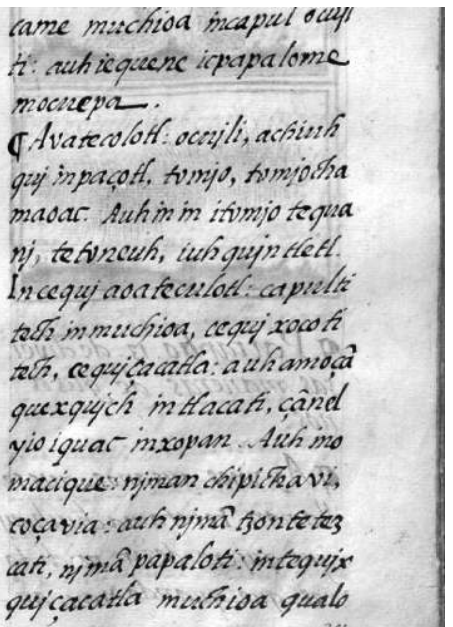

FIG. 15 - Chenille velue venimeuse nommée Auatecolotl [Códice Florentino 1979, XI, f. 103r]. 
Les effets induits par les piqûres des chenilles de Mégalopyges et ceux d'Automeris étant similaires, il est possible que le même terme ait été appliqué, en nahuatl, à ces deux types de chenilles, l'une aux " plumes » duveteuses blanches (Mégalopyges), l'autre aux « plumes » vertes (Automeris) et qu'il faudrait associer aux deux formes prises par les « serpents à plumes » de Teotihuacan, l'une étant un serpent aux plumes blanches, l'autre un ophidien aux plumes vertes comme sur la peinture murale dite des « animaux mythologiques » (cf. Figure 16 et II dans le cahier en couleurs).

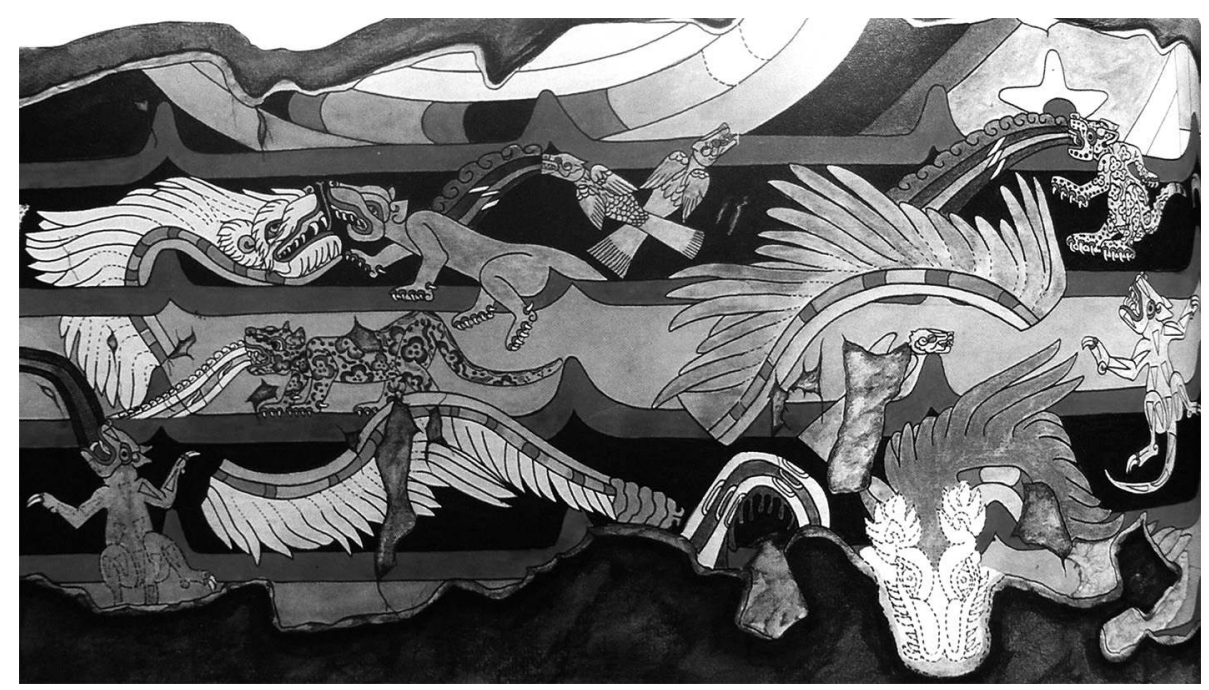

FIG. 16 et II dans le cahier en couleurs - Peinture murale dite des « animaux mythologiques » sur laquelle apparaissent des serpents à plumes de couleurs verte et blanche [C Consejo Nacional para la Cultura y las Artes, Instituto Nacional de Antropología e Historia, Mexico, d'après B. de la Fuente 1995-1996].

Toujours à propos des chenilles d'Automeris, Beutelspacer les associe à une autre citation de Fernández de Oviedo y Valdés :

« Del Temahoani. Es un gusano negro erizado de pequeñas espinas amarillas que están dispuestas apretadamente en series esparcidas y que son dañinas y venenosas. Vive en Tepoztlán, donde algunos lo llaman xuxhuiahotecólotl (C.VII). »

"Hace referencia a orugas urticantes de la familia Saturniidae, probablemente del género Automeris. También se le llamó temauani o temahua, y significa "el que contagia o comunica una enfermedad" (de maua, contagiar un mal). » (Beutelspacher 1988, p. 22)

Il s'agit en fait de la même espèce, Automeris sp., que celle évoquée au début de cet article. Nous faisions remarquer que la référence au hibou ou à la chouette, 
dans l'analyse étymologique du terme nahuatl qui pourrait la désigner, viendrait plutôt du fait qu'elle possède sur ses ailes, à l'état d'imago, des ocelles qui rappellent des yeux de chouettes ou de rapaces. "Hibou-épineux-des-fleurs », « Hibou-urticant-des-fleurs », traduction littérale de Xochiautecólotl-ou encore «Hibou-épines-en-fleur (touffe, plumeau)» selon Chamoux (communication personnelle, juin 2012) - serait une désignation métaphorique de la chenille d'Automeris et de sa forme adulte (Figure 17). L'autre terme qui la désigne Temahoani « celui qui communique une maladie » serait plus une référence aux effets induits qu'à la forme de la chenille. L'ensemble des espèces nommées ici cible la même classe de chenilles urticantes désignée sous le terme chinalahautl en nahuatl (« derrière-épineux »), mais dont certaines peuvent être désignées grâce au terme coatl.

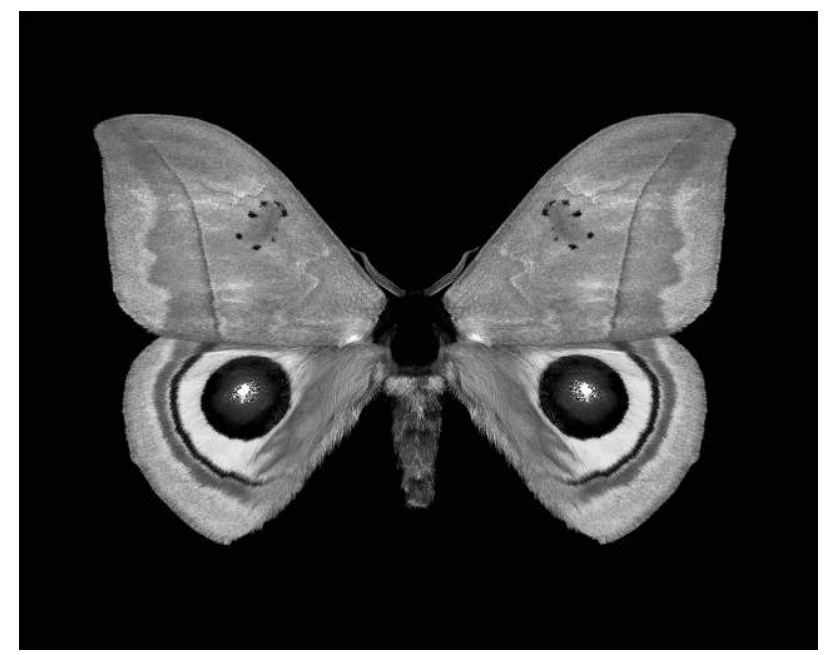

FIG. 17 - Forme adulte d'Automeris $s p$. (femelle). En association avec sa forme larvaire urticante, le mimétisme des ocelles et des ailes avec une face de hibou donnent raison de son nom en nahuatl « Hibou-épineux-des-fleurs », ou « Hibou-à-épines-en-fleur » (Xochiautecolotl) [d'après http://tomboyyobmot.blogspot.fr/2010/11/mothy-and-butterfilled.html].

Dans la famille des Saturnidés, les chenilles qui, par analogie, s'apparentent le plus aux figurations de serpents à plumes sont donc celles des Automeris et des Lonomia (toutes deux présentes en Mésoamérique, bien que les variétés les plus virulentes de Lonomia soient plutôt sud-américaines). Or ces deux espèces subissent d'importantes variations de couleurs durant leur cycle de croissance. Surtout, elles possèdent des traits anatomiques que l'on peut rapprocher de certaines images des serpents à plumes comme, par exemple, le fait qu'elles ont une tête de couleur bleue turquoise et deux bandes de couleurs caractéristiques, 
une rouge au dessus d'une jaune, qui les parcourent de tout leur long. Dans les illustrations des codex (comme celle de la page 14 du Codex Borbonicus: Figure 18 et I bas dans le cahier en couleurs), l'iconographie du serpent à plumes reprend cette caractéristique en présentant ce dernier avec deux bandes, une blanche ou jaune en dessous d'une rouge, parcourant son corps de tout son long à la façon de la chenille (ce même mode chromatique a été adopté dans la figuration du serpent à plumes du folio $18 \mathrm{r}$ du Codex Telleriano-Remensis et de la planche 67 du Codex Borgia). Dans l'image citée du Codex Borbonicus, la figure du serpent à plumes est accompagnée d'un petit serpent à deux têtes. La forme que lui a donnée l'artiste aztèque est celle d'une chenille " arpenteuse » de la famille des Géométridés, nommée tlatamacihauni, "arpenteur » en nahuatl ( $c f$. Beutelspacher 1988, p. 21), suivant une analogie de son mode de déplacement avec l'écartement du pouce et de l'index de «l'arpenteur» évoluant sur une distance à mesurer : le corps de la chenille rapproche ainsi une extrémité de l'autre, ce qui donne au milieu de son corps une courbure caractéristique. Ce " serpent » à deux têtes est probablement la figuration imagée d'une chenille qui « mord » par les deux extrémités. Enfin, ses têtes sont de couleur turquoise, ce qui donne une indication précieuse de l'espèce représentée, puisque les chenilles urticantes des espèces-sources de Quetzalcóatl sont dotées d'une tête et de l'arrière de leur corps de la même couleur. Cette figure semble ainsi être un indicateur que le « serpent à plumes » serait en réalité une chenille.

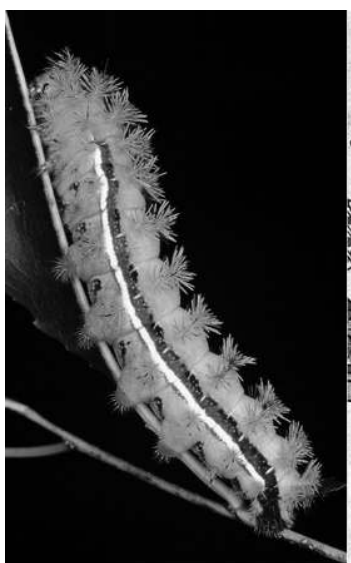

a.

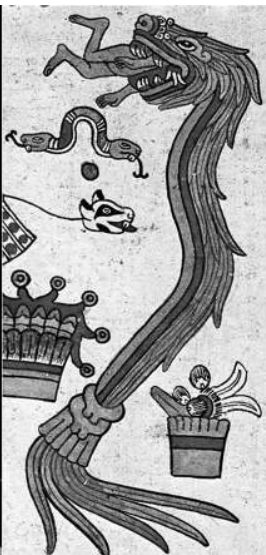

b.

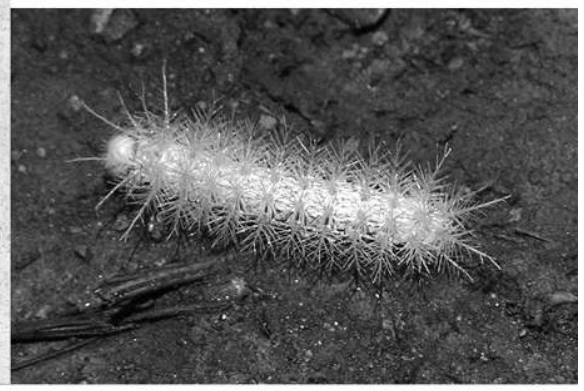

c.

FIG. 18 et I bas dans le cahier en couleurs - Comparaison entre des chenilles d'Automeris io et la figuration du serpent à plumes à la page $14 \mathrm{du}$ Codex Borbonicus : on notera la présence de bandes jaune et rouge parcourant les corps de la chenille et du serpent fantastique de façon analogue ainsi que la tête de couleur turquoise de certains exemplaires

[Fig. 18a : d'après http://www.discoverlife.org/mp/20q?search=Automeris+io ;

Fig. 18b: Codex Borbonicus p. 14; Fig. 18c: d'après http://en.wikipedia.org/wiki/File: Hairy_caterpillar_\%28Costa_Rica\%29.jpg]. 
Le nez, ou museau de l'être fantastique est peint de turquoise, tout comme ses yeux, ainsi qu'une partie de la structure vibrante des serpents à sonnette dont il est affublé. Or nous avons vu que le « nez » du « monstre », c'est-à-dire l'image mimétique que renvoie la chenille, correspond à sa tête véritable, de couleur bleue, et donc que les parties de sa tête où se trouvent ses yeux, également de couleur turquoise, sont rendues sur le serpent à plume sous la forme d'yeux rehaussés de cette couleur. Enfin, en plus des structures urticantes de couleur verte du corps de la chenille, (ses « plumes »), celle-ci est dotée de plusieurs excroissances urticantes de couleur turquoise, disposées en arrière de la tête à la façon d'une coiffe en éventail, ou collerette, dirigée vers l'avant. Ce trait anatomique pourrait expliquer que les têtes du serpent à plume en pierres taillées du temple du Serpent à plumes à Teotihuacan soient dotées de telles collerettes. Sur la chenille, ces mêmes structures turquoises se retrouvent à l'arrière du corps où elles se réduisent parfois, suivant les variétés d'Automeris, à deux pics en forme de crochet. Hautement urticantes, les chenilles d'Automeris io, dont l'iconographie de Quetzalcóatl semble s'inspirer fortement, ressemblent à celles de Lonomia electra dont le contact est souvent mortel pour l'homme et qui possèdent un corps légèrement plus noir qu'Automeris (bien qu'Automeris zephyria ait un corps noir). Cette propriété létale de la chenille devrait être rapprochée du terme donné dans la citation de Fernández de Oviedo y Valdés, temahoani «transmettre un mal », mais aussi peut-être du fait que le serpent à plumes de quetzal est souvent figuré dans les codex aztèques dévorant un être humain.

L'empoisonnement de Lonomia electra, variété mésoaméricaine des représentants de cette espèce dont les plus virulentes se trouvent essentiellement en Amérique du Sud, est analogue, dans ses effets, à celui d'une morsure de serpent à sonnette. Les réactions les plus immédiates de ce qui est aujourd'hui connu comme l'empoisonnement de Lonomia (lonomiasis) sont des saignements de la zone touchée, dus à la présence d'un anticoagulant au sein des structures urticantes agissant comme de véritables rasoirs, des douleurs intenses, de la fièvre et des vomissements. Selon plusieurs publications spécialisées, le venin propagé par cette chenille est tout simplement le plus violent au monde... (en termes de ratio : une quantité mille fois inférieure à celle produite par une morsure de serpent à sonnette produit des effets analogues sur un organisme humain). Il est vraisemblable que la présence d'une queue de serpent à sonnette en association avec le serpent à plumes de quetzal dans les représentations mésoaméricaines ait eu pour origine la similitude des réactions aux piqûres de ces chenilles avec une morsure de crotalidé.

Dans les cas ayant entraîné la mort suite à un contact avec plusieurs chenilles - grégaires à leur premier stade de développement, elles forment des amas radiaux sur les troncs d'arbres -, les causes sont essentiellement dues aux hémorragies internes qui prennent le pas sur les saignements externes à partir des muqueuses et sur les vomissements de sang: ainsi, la mort n'intervient que 
plusieurs heures, voire plusieurs jours après l'empoisonnement. Si l'iconographie teotihuacan du serpent à plumes, mais aussi d'autres animaux comme les jaguars, loups et pumas, figure des scènes sanglantes, soit qu'un couteau sacrificiel serve de langue au serpent, soit qu'il soit montré vomissant lui-même du sang, ou placé auprès de cœurs de sacrifiés obtenus par cardioectomie, et si l'animal-source de ces représentations est une chenille urticante, alors cette iconographie doit être interprétée dans le sens d'une mise en image des effets causés par des êtres qui, comme la chenille de Lonomia electra, produisent des écoulements de sang, on pourrait ainsi parler de la figuration des effets létaux et du choc hémophilien occasionnés par son contact.

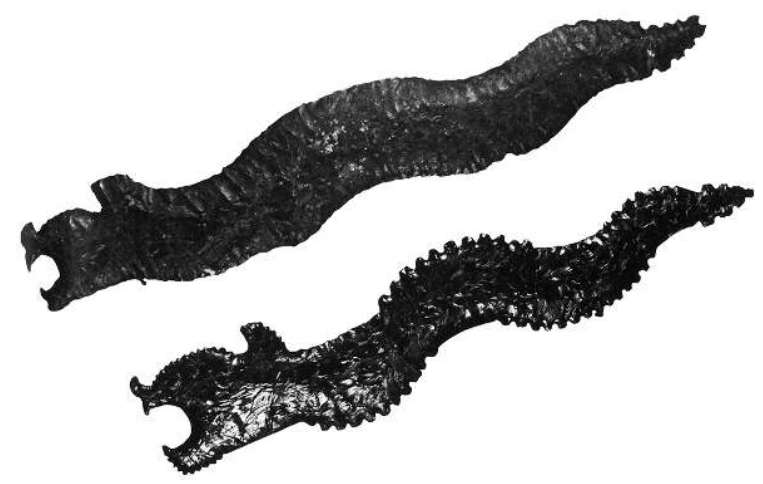

FIG. 19 - Excentriques d'obsidienne en forme de serpents (Pyramide de la Lune, Teotihuacan) [C Consejo Nacional para la Cultura y las Artes, Instituto Nacional de Antropología e Historia, Mexico, photo Martirene Alcantara].

Quant aux excentriques en obsidienne à forme de serpents trouvés dans les dépôts de fondation de la Pyramide de la Lune à Teotihuacan (Cabrera et Sugiyama 2009, p. 67), ils évoqueraient aussi les chenilles : de fait, ils sont une mise en situation, grâce à un artéfact, de ce que ce dont ces larves sont la cause lorsqu'elles entrent en contact avec une peau humaine : provoquer des saignements ( $c f$. Figures 19 et 20).

Dans ces dépôts, en plus d'une indication des axes directionnels du plan horizontal, il faudrait s'interroger sur la similitude donnée à la disposition radiale des excentriques (Figure 20). En effet, la disposition radiale, tête tournée vers la périphérie, des couteaux-serpents d'obsidienne, semble reprendre celle qu'adoptent les chenilles de ces espèces urticantes lorsqu'elles forment un amas grégaire sur un tronc d'arbre. Il resterait à comprendre en quoi la disposition des excentriques d'obsidienne en forme de serpents, analogue à celle des chenilles, porterait en elle une valeur explicative pour leur présence auprès d'individus sacrifiés. 


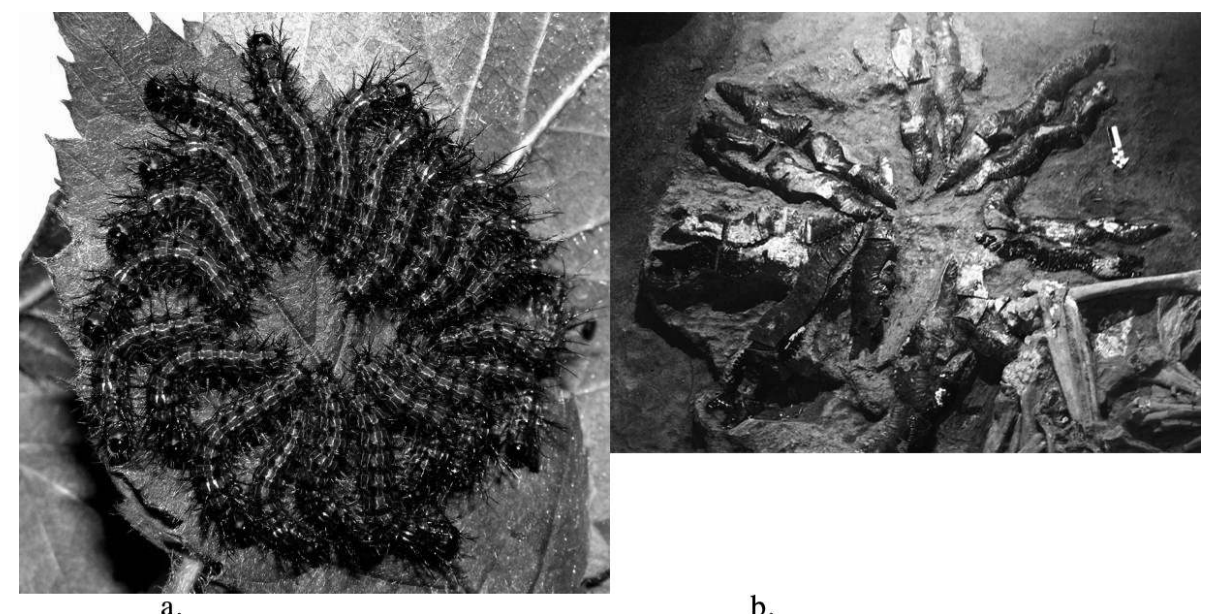

FIG. 20a et 20b - Amas radial de chenilles de Lonomia electra et disposition radiale d'excentriques d'obsidienne (dépôt-offrande $\mathrm{n}^{\circ}$ 6, Pyramide de la Lune, Teotihuacan) [Fig. 20a : d'après http://www.entomologenportal.de/g4297p11130-Lonomia-electra.html ; Fig. 20b : Cabrera et Sugiyama 2009].

\section{Conclusion}

La figure des serpents fantastiques dans l'univers représentationnel mésoaméricain doit être mise en parallèle avec la place occupée, dans ce même univers, par les lépidoptères et permettre ainsi de reconnaître que ces « serpents » sont une mise en images des parties que constituent des êtres réels, à savoir les formes larvaires des papillons. La permanence dans le temps de ces traits, dans les différentes sociétés précolombiennes du Mexique, montre que le processus de perception de l'environnement en tant que «lecture analogique», propre à l'esprit humain, est la marque, dans cet espace culturel, d'une attention particulière aux formes signifiantes apparaissant au sein d'êtres ou d'espèces qui servent dans les processus de nomination et/ou dans la création des êtres mythologiques. Si la figure mythologique du serpent à plumes, qui donnera naissance au Quetzalcóatl du Postclassique, se construit à partir de la chenille hautement urticante d'un papillon de nuit, Automeris io qui, sous sa forme adulte, offre un mimétisme construit sur l'image d'une face de chouette ou de jaguar et que ces derniers animaux se retrouvent combinés dans des artéfacts à forme de papillon, comme dans le cas de la coiffe des urnes zapotèques par exemple, alors c'est l'ensemble des formes culturelles de cette aire qu'il faut réinterroger au regard de ce constat.

Des figures de l'iconographie de Teotihuacan telles les «conques emplumées », les « jaguars réticulés », les pumas et coyotes « à plume » devraient être 
examinées, en prenant en compte les principes dégagés ici. L'opération mentale de composition étudiée dans cet article montre qu'elle nécessite une certaine indépendance vis-à-vis du sujet percevant. La prise de conscience n'intervient que dans un second temps, c'est-à-dire au moment où l'image portée sur une espèce donnée apparaît à l'observateur ; lorsqu'à regarder une chenille apparaît un serpent.

Se pose ensuite la question de l'origine du surgissement mental de ces images : l'imputation d'une qualité d'agent résidant dans la chose perçue (le serpent qui se manifeste en image sur la chenille et, en esprit, " dans » celle-ci) devient alors une modalité explicative possible de la part des cultures mésoaméricaines. Il s'agit là d'une forme d'ethno-ontologie distincte de la nôtre, puisque cette dernière postule, en quelque sorte, le chemin inverse. Pour l'ontologie naturaliste en effet, ce phénomène, bien qu'inscrit sur les corps des lépidoptères, trouve son origine dans les mécanismes perceptifs - donc mentaux - du sujet percevant. *

* Manuscrit reçu en décembre 2012, accepté pour publication en février 2014.

\section{Notes}

Les idées principales de ce texte ont été exposées en novembre 2009 dans le cadre de l'atelier "Chimères, images polarisées et rituels de transformation », coordonné par Guilhem Olivier et Johannes Neurath (GDRI : Anthropologie et histoire des arts, Musée du quai Branly). Nous voudrions remercier tout particulièrement les coordinateurs de cette journée pour la relecture de ce texte et exprimer ici notre gratitude aux évaluateurs du Journal de la Société des Américanistes pour les précieux conseils et compléments d'information qu'ils ont eu la générosité de nous communiquer.

1. Pour la Mésoamérique, cette image « en double » n'est pas sans reposer les problèmes soulevés par des conceptions comme le tonalisme et le nahualisme. Dans la première, il s'agit de l'une des facettes de la personnalité du sujet ; pour la deuxième, d'un double indépendant de ce dernier. De façon plus générale, le terme " nahualli » était aussi employé comme synonyme de " déguisement », un aspect que revêtent les êtres et les choses, mais qui semblerait avant tout être lié à l'image mentale que chaque être est en mesure de produire auprès d'observateurs humains.

2. Même s'il reste une incertitude quant au passage par l'image d'une forme naturelle, la forme du bec permet l'identification de son appartenance ou non à un oiseau de proie nocturne (cf. Chomel de Coelho 1975, p. 330).

3. Les papillons ne «font » rien coïncider volontairement, puisqu'il ne s'agit ici que d'une forme résultant des processus de sélection et d'adaptation naturelles : l'image mimétique est « façonnée » par les prédateurs de papillons qui laissent s'échapper les exemplaires qui ressemblent le plus à leurs prédateurs (petits félins pour les oiseaux, chouettes, rapaces et serpents pour les lézards) et génèrent ainsi l'image de leurs prédateurs sur leurs proies.

4. Peinture murale de la Zone 5a, pièce 7, in Miller 1973, p. 86, figs. 135 et 136 ; De la Fuente 1995-1996, vol. 1, p. 77, fig. 6.13.

5. Les pages du Livre XI, cap. 5, § 12 de Sahagún (1950-1981, XIII, pp. 98-100) sont consacrées aux chenilles : "monoquia ahzo tzoncōāmeh ahnōzo ocuiltin » : on évacue par l'anus les (vers appelés) tzoncoatl ou les vers (en général) ocuilin.

6. Dans le Livre XI, cap. 12, § 8, (Sahagún 1950-1981, p. 269), il est dit que les anciens appelaient cōātl le chemin, " ohtli' », d'où l'expression : " in ìohhuitzin in ìcōātzin totēcuihyo », " le chemin de notre Seigneur ». Anderson et Dibble traduisent : « it is a little danger, a little serpent of our lord». 
7. Historia general y natural de las Indias, ca 1545.

8. Voir aussi Karttunen (1992, p. 142), pour cette même association entre chenille et l'analogie « serpent-cerf ».

9. Nous reviendrons plus loin sur les poils blancs des chenilles de Mégalopyges, dans la mesure où certains serpents fantastiques sont représentés avec des plumes blanches dans les peintures murales de Teotihuacan.

10. Il en va ainsi de cette devinette en nahuatl : « iztac tetzintli quetzalli commanticah » : « une petite pierre blanche, elle porte des plumes de quetzal » qui désigne l'oignon. Les fanes vertes de l'oignon sont comparées à des plumes de quetzal. Sahagún (1950-1981, VI, p. 238) compare l'oignon soit à une pierre, soit à une tête, munie de plumes de quetzal (Wimmer 2006) [http://sites.estvideo.net/malinal/ x/nahuatlXOMP.html\#XONACATL].

\section{RÉFÉRENCES CITÉES}

BerLo Janet C.

1983 «The warrior and the butterfly: central Mexican ideologies of sacred warfare and Teotihuacan iconography », in Janet C. Berlo (éd.), Text and image in Pre-columbian art : essays on the interrelationship of the verbal and visual arts, Archaeopress, coll. « British Archaeological Reports, International series »180, Oxford, pp. 79-118.

BERRIN Kathleen (éd.)

$1988 \quad$ Feathered serpents and flowering trees : reconstructing the murals of Teotihuacan, The Fine Arts Museums of San Francisco, San Francisco.

Beutelspacher Carlos R.

1988 Las mariposas entre los antiguos mexicanos, Fondo de Cultura Económica, Mexico.

Cabrera C. Rubén et Saburo Sugiyama

2009 "Fouilles de la pyramide de la Lune », in Teotihuacan, cité des dieux, Musée du quai Branly/Somogy éditions d'Art, Paris, pp. 58-67.

Chomel de Coelho Martine

1975 «Recapitulación y precisión sobre la identificación de la lechuza en Teotihuacan ", in XLI Congreso Internacional de Americanistas, Actas, II, Instituto Nacional de Antropología e Historia, Mexico, pp. 323-333.

Codex Borbonicus

Voir FAMSI [http://www.famsi.org/research/graz/borbonicus/thumbs_ $0 . \mathrm{html}]$

Codex Borgia

1993 The Codex Borgia : a full-color restoration of the ancient Mexican manuscript, édité par Gisele Díaz et Alan Rodgers, Dover publications, New York.

Codex Telleriano-Remensis

(Loubat 1901): Voir FAMSI [http://www.famsi.org/research/loubat/ Telleriano-Remensis/thumbs0.html] 


\section{Códice Florentino}

1979 Edición facsímil, Secretaría de Gobernación, México.

Voir Sahagún Fray Bernardino de (1950-1981).

DARLington H. S.

1931 "The "fire-snakes" of the Aztec calendar stone », Anthropos, 26 (5/6), pp. 637-646.

De la Fuente Beatriz (éd.)

1995-1996 La pintura mural prehispánica en México. I, Teotihuacan, 2 vol., Universidad Nacional Autónoma de México, Instituto de Investigaciones Estéticas, Mexico.

Descola Philippe

2005 Par-delà nature et culture, Gallimard, coll. "Bibliothèque des sciences humaines », Paris.

\section{ENCISO Jorge}

1947 Sellos del antiguo México, Instituto Nacional de Antropología e Historia, Mexico.

Franco José Luis

1955 «Representaciones de la mariposa en Mesoamérica », El México Antiguo, 9, pp. 195-244.

\section{KARADIMAS Dimitri}

2010 "Animaux imaginaires et êtres composites", in Catalogue de l'exposition "La Fabrique des images. Visions du monde et formes de la représentation », Musée du quai Branly/Somogy éditions d'Art, Paris, pp. 184-191.

\section{KartTUNEN Frances}

1992 An analytical dictionary of Nahuatl, Universtity of Oklahoma Press, Norman.

LÉvi-STRauss Claude

1963 La Pensée sauvage, Plon, Paris.

LÓPEZ LuJÁN Leonardo

1991 «Peces y moluscos en el libro undécimo del Códice Florentino », in Oscar J. Polaco (éd.), La fauna en el Templo Mayor, Instituto Nacional de Antropología e Historia, Proyecto Templo Mayor, Mexico, pp. 213-263.

Miller Arthur G.

1973 The mural painting at Teotihuacan, Dumbarton Oaks, Trustees for Harvard University, Washington, D. C

Molina Alonso de

1966 Vocabulario en lengua castellana y mexicana, édité par Antonio de Spinosa, Mexico [1571]. 
NichOLSON Henry B.

2000 "The iconography of the feathered serpent in Late Postclassic Central Mexico ", in Davíd Carrasco, Lindsay Jones et Scott Sessions (éd.), Mesoamerica's Classic heritage, from Teotihuacan to the Aztecs, University Press of Colorado, Boulder, pp. 145-164.

RAGOT Nathalie

2000 Les au-delàs aztèques, Archaeopress, coll. "British Archaeological Reports, International series 881/Paris monographs in American archaeology » 7, Oxford.

SAHAGÚN Fray Bernardino de

1950-1981 Florentine Codex. General History of the things of New Spain, Fray Bernardino de Sahagún, éd. et trad. par Charles E. Dibble et Arthur J. O. Anderson, The School of American Research and the University of Utah, Santa Fe.

SÉJOURNÉ Laurette

1962 El universo de Quetzalcóatl, Fondo de Cultura Económica, Mexico.

Sellen Adam T.

2007 El cielo compartido. Deidades y ancestros en las vasijas efigie zapotecas, UNAM/Centro Peninsular en Humanidades y Ciencias Sociales, coll. «Monografías » 4, Mérida.

SugiYama Saburo

2000 《Teotihuacan as an origin for Postclassic feathered serpent symbolism », in Davíd Carrasco, Lindsay Jones et Scott Sessions (éd.), Mesoamerica's Classic heritage, from Teotihuacan to the Aztecs, University Press of Colorado, Boulder, pp. 117-143.

TAUBE Karl

2000 "The turquoise hearth. Fire, self sacrifice, and the central Mexican cult of war ", in Davíd Carrasco, Lindsay Jones et Scott Sessions (éd.), Mesoamerica's Classic heritage, from Teotihuacan to the Aztecs, University Press of Colorado, Boulder, pp. 269-340.

2002 «La serpiente emplumada en Teotihuacan», Arqueología Mexicana, 9 (53), pp. 36-41.

THOUvenot Marc

1982 Chalchihuitl. Le jade chez les Aztèques, Institut d'ethnologie/Musée de l'Homme, Paris.

VAN DiNTER M. H.

2006 Tribal tattoo designs from the Americas, Mundurucu, Amsterdam.

WiMmer Alexis

2006 Dictionnaire de la langue nahuatl classique [http://sites.estvideo.net/ malinal/nahuatl.page.html] 